This work has been submitted to the IEEE for possible publication.

Copyright may be transferred without notice, after which this version may no longer be accessible 


\title{
Influence of Magnetic Scaffold Loading Patterns on their Hyperthermic Potential against Bone Tumors
}

\author{
Matteo B. Lodi†, Graduate Student Member, IEEE, Nicola Curreli ${ }^{\dagger}$, Sonia Zappia, Luca Pilia, \\ Maria Francesca Casula, Sergio Fiorito, Ilaria Catapano, Francesco Desogus ${ }^{*}$, Teresa \\ Pellegrino, Ilka Kriegel, Lorenzo Crocco, Senior Member, IEEE, Giuseppe Mazzarella, \\ Senior Member, IEEE, Alessandro Fanti*, Member, IEEE
}

\begin{abstract}
Magnetic scaffolds have been investigated as promising tools for the interstitial hyperthermia treatment of bone cancers, to control local recurrence by enhancing radio- and chemotherapy effectiveness. The potential of magnetic scaffolds motivates the development of production strategies enabling tunability of the resulting magnetic properties. Within this framework, deposition and drop-casting of magnetic nanoparticles on suitable scaffolds offer advantages such as ease of production and high loading, although these approaches are often associated with a non-uniform final spatial distribution of nanoparticles in the biomaterial. The implications and the influences of nanoparticle distribution on the final therapeutic application have not yet been investigated thoroughly. In this work, poly-caprolactone scaffolds are magnetized by loading them with synthetic magnetic nanoparticles through a drop-casting deposition and tuned to obtain different distributions of magnetic nanoparticles in the biomaterial. The physicochemical properties of the magnetic scaffolds are analyzed. The microstructure and the morphological alterations due to the reworked drop-casting process are evaluated and correlated to static magnetic measurements. $\mathrm{THz}$ tomography is used as an investigation technique to derive the spatial distribution of nanoparticles. Finally, in silico multiphysics experiments are used to investigate the influence on the loading patterns on the interstitial bone tumor hyperthermia treatment.
\end{abstract}

Index Terms-bone tumors, hyperthermia, magnetic nanoparticles, scaffolds, THz tomography

M. B. Lodi, A. Fanti, G. Mazzarella are with the Department of Electrical and Electronic Engineering, University of Cagliari, via Marengo 2, 09123, Cagliari, Italy (corresponding e-mail: alessandro.fanti@diee.unica.it).

N. Curreli, S. Fiorito, T. Pellegrino, I. Kriegel are with the Istituto Italiano di Tecnologia, via Morego 30,16163 Genoa, Italy.

S. Zappia, I. Catapano, L. Crocco are with the Institute for the Electromagnetic Sensing of the Environment, National Research Council of Italy, 80124 Naples, Italy.

L. Pilia, M. F. Casula, F. Desogus are with the Department of Mechanical, Chemical and Materials Engineering, University of Cagliari, via Marengo 2, 09123, Cagliari, Italy (corresponding email: f.desogus@dimcm.unica.it).

${ }^{\dagger}$ M. B. Lodi and N. Curreli contributed equally to this work.

\section{INTRODUCTION}

$\mathrm{H}$ YPERTHERMIA is a powerful thermal therapy used in oncology [1]-[4]. Hyperthermia treatment (HT) aims to rise the temperature of a target tissue in the range $40-44^{\circ} \mathrm{C}$, for at least 30-60 min, in order to direct damage the DNA, hamper its repair and replication at the cellular level [4]. Effects reported during heat delivery include free radicals formation, cellular protein denaturation, extracellular $\mathrm{pH}$ increase, and occurrence of hypoxic environment [3]. HT can enforce immunomodulation, by boosting the immune system response (e.g., by upregulating the heat shock protein release) [2]. However, the full clinical potential of HT occurs when used in synergy with other treatment modalities, such as chemotherapy and radiotherapy [3], [5]. The temperature raise increments the blood perfusion at the tumor site, enhancing the cellular permeability to drugs (e.g., paclitaxel, doxorubicin) [4]. Temperature elevations acts also as a powerful radiosensitizer, opening new therapeutic routes for several problematic neoplasms [3], [6].

Primary and secondary bone cancers are a class of pathologies which can strongly benefit from hyperthermia [7][9]. Osteosarcomas (OS) are aggressive, have high-recurrence $(40 \%)$ and metastatic $(20 \%)$ rates and are also radio-resistant bone tumors [7]. Currently, surgical resection is the goldstandard approach [7], [8]. However, the surgery consists in amputation or limb-sparing tumor resection, resulting in disabling outcomes [8], [9]. Alternative strategies are studied, e.g., cryotherapy or embolization, but they are still experimental [7]. Recently, the clinical outcomes of adjuvant biophysical therapies, especially HT, are gaining the interest of scientific community [10]. Microwave hyperthermia, performed by placing interstitial antennas in the surgical bed, was investigated and, compared to limb amputation, resulted in marginal differences as standalone treatment modality [11][14], while reducing the local recurrence rate of more than $20 \%$, when combined to other therapies [3]. Despite the functional and quality-of-life advantages, the microwave treatment of bone tumors has the post-operative drawback of a high fracture rate of about $30 \%$, ascribed to bone weakening [11], [12]. Besides the aforementioned specific limitations of 


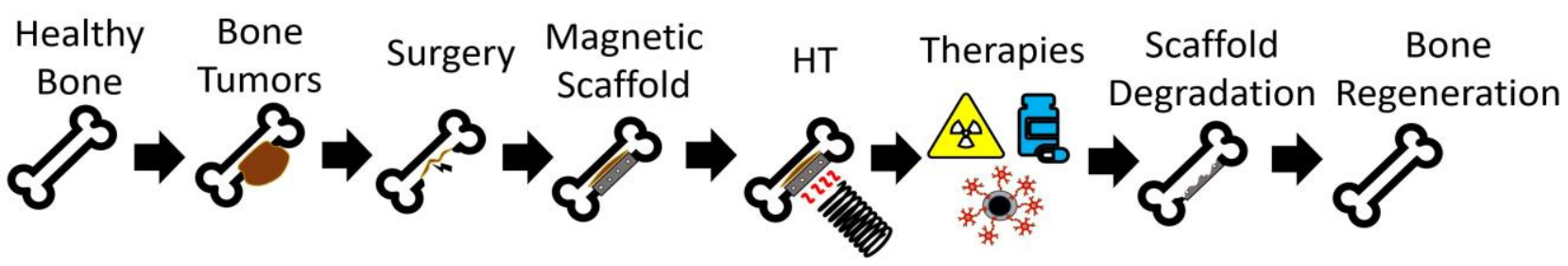

Fig. 1. Magnetic Scaffolds as theranostic system: after the insurgence of a bone tumor, surgery is carried out and the bone gap can be filled with a magnetic prosthetic implant. Then hyperthermia treatment $(\mathrm{HT})$ is performed by applying a radiofrequency magnetic field of clinical use. $\mathrm{HT}$ has direct toxic effects on the cells and has the potential of enhancing radiotherapy and chemotherapy effectiveness. After local recurrence is controlled, the magnetic scaffold can be used as a tool for accelerating bone repair and regeneration.

surgery and microwave hyperthermia, both approaches require a graft or a biomaterial, i.e., a scaffold, for post-operative management.

By combining the strict anti-cancer therapeutic and the postoperative orthopedic requirements, the technological advancements in hyperthermia delivery lead to the innovative idea of manufacturing nanostructured magnetic biomaterials to be used as implanted thermo-seeds to perform local, interstitial HT of bone cancers by applying an external radio-frequency (RF) field [15]-[24], as shown in Fig. 1. A so-called magnetic scaffold (MagS) can be obtained by chemical doping of bioceramic or physical loading of magnetic nanoparticles (MNPs) in a polymeric matrix to obtain a multifunctional and theranostic device [15]-[27]. When exposed to a time-varying magnetic field, the MNPs embedded in the implanted biomaterial dissipate magneto-energy into heat, which is transferred by conduction to the surrounding pathologic tissues [17], [21]. Moreover, as shown in Fig. 1, after the elimination of the residual cancer cells upon control of local recurrence, MagS could be used to regulate and enhance the bone healing process (e.g., by controlling $\mathrm{Ca}^{2+}$ ion fluxes or through the mechano-transduction of exogenous magnetic stimuli) [28]-[34], or by allowing magnetic resonance imaging (MRI) for tissue healing and implant monitoring [35], [36].

To date, magnetic hydrogels were obtained by blending procedure to include ferrite (e.g., $\gamma-\mathrm{Fe}_{2} \mathrm{O}_{3}, \mathrm{Fe}_{3} \mathrm{O}_{4}, \mathrm{CoFe}_{2} \mathrm{O}_{4}$ ) MNPs within the scaffold [16], resulting in moderate saturation magnetization $\left(M_{S}\right)$ values (ranging from 0.1 to 11 emu/g). MagS with more pronounced magnetic properties $\left(M_{S}\right.$ = 1-10 emu/g) were obtained by sol-gel techniques, coprecipitation, hydrothermal process or other chemical routes to obtain intrinsically magnetic hydroxyapatite [37], or $\beta$ tricalcium phosphate [17], [38] modified hardystonite $\left(\mathrm{Ca}_{2} \mathrm{ZnSi}_{2} \mathrm{O}_{7}\right)$ [24] or bredigite $\left(\mathrm{Ca}_{7} \mathrm{Mg}\left(\mathrm{SiO}_{4}\right)_{4}\right)$ scaffolds [39]. For this kind of MagS, the amount of magnetic crystalline phase, the final microstructure, and the particle's interactions are key factors in determining the hyperthermia potential [17], [21]. The tuning and controllability of these parameters can be rather complex, and the process synthesis can be relatively expensive [21]. In this context, the use of polymeric matrix for MagS was investigated. Electrospinning of chitosan, poly(lactic acid) (PLA), polyvinyl alcohol (PVA), and silk fibroin was performed to obtain magnetic, quasi-3D nanofibrous structures, with a $M_{s}$ of about $2-8 \mathrm{emu} / \mathrm{g}$, but limited hyperthermia and regenerative potential [40], [41]. A 3D printed magnetic nanocomposite scaffold made of polycaprolactone (PCL) filament loaded with $\mathrm{Fe}_{3} \mathrm{O}_{4}$ MNPs demonstrated to be capable of raising the temperature of $5^{\circ} \mathrm{C}$ in $5 \mathrm{~min}$ when exposed to a field intensity of $15 \mathrm{mT}$ and at a frequency of $293 \mathrm{kHz}$ [42]. The functionalization with MNPs can be carried out on more complex, biomimetic scaffold geometries following dip-coating or ferrofluid impregnation procedures [40], [43]-[47].

The technique consists of making a colloidal suspension of MNPs which is physisorbed by capillarity on the surface defects and inhomogeneities of the biomaterial. With these methods, a hybrid collagen-hydroxyapatite matrix can be loaded with $1-5 \%$ of MNPs, under sonication, and reach 15 $\mathrm{emu} / \mathrm{g}$ if the procedure is repeated multiple times [45]. Similarly, silk fibroin scaffold can be magnetized successfully by ferrofluid, showing an $8^{\circ} \mathrm{C}$ increase in about $100 \mathrm{~s}$, under the action of a $30 \mathrm{mT}$ and $293 \mathrm{kHz}$ magnetic field [47]. However, it is worth to highlight that the impregnation procedures do not guarantee a uniform dispersion of the MNPs in the scaffold matrix, as underlined by the static magnetic measurements of [43], which measured a $20 \%$ variation of the saturation magnetization in about $5 \mathrm{~mm}$. Diphasic distribution of maghemite MNPs in polymers was studied [44], with poor reasoning on the design and implications. Overall, state-ofthe-art analysis [43]-[47] highlight that the influence of how the loading pattern could affect the MagS performance was investigated only from a numerical point of view [48]. As a matter of fact, the tuneability and controllability of the production process have never been investigated in relation to the functional properties and final applications. Since MagS can be interpreted as a novel, more bio-compatible version of steel thermo-seeds used for the treatment of deep-seated tumors [17], [21], [49], [50], they are constrained to the highquality assurance criteria of interstitial hyperthermia [51]. It is therefore mandatory to investigate, from an engineering point of view, how and if different loading patterns could hamper the hyperthermia treatment of bone tumors.

In this work we aim to investigate if and how the magnetization degree and loading pattern of magnetic scaffolds can influence the outcome of the hyperthermia treatment of bone tumors. To this aim, the design and production of magnetic scaffolds was achieved by controlled 


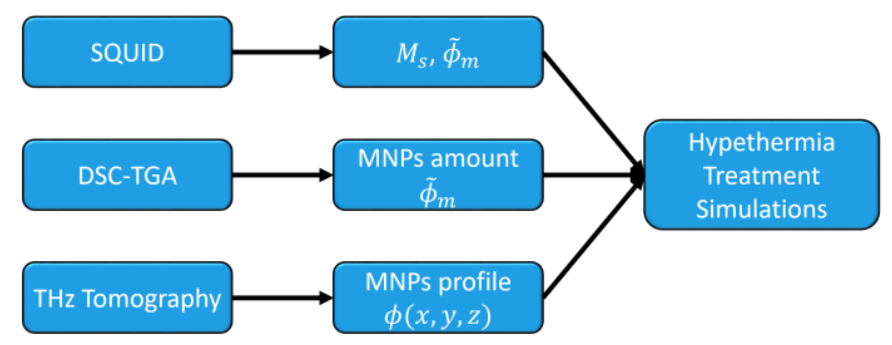

Fig. 2. The physiochemical characterization of magnetic scaffolds aims to extract the magnetic properties of the samples through the analysis of the saturation magnetization MS, derive the maximum amount of magnetic nanoparticles with DSC-TGA measurements $\left(\tilde{\phi}_{m}\right)$, and retrieve the nanoparticles profile inside the biomaterial, $\phi(x, y, z)$. This information is used to simulate the hyperthermia treatment with the magnetic scaffolds from Fig. 3 and study the influence of nanoparticles' pattern on the quality of treatment.

deposition of ferrite magnetic nanocrystals (MNCs) on commercial PCL scaffolds featuring a $90^{\circ}$-shifted highly porous mesh. As summarized by Fig. 2, the manufactured magnetic scaffolds are characterized in terms of static magnetic response. The amount of magnetic phase and thermal stability are assessed through differential scanning calorimetry and thermogravimetric analysis. The morphological investigation is carried out by electron microscopy and the spatial distribution of the MNCs in the scaffold volume is retrieved by $\mathrm{THz}$ tomography. As shown in Fig. 4, by combining the magnetic properties and the loading patterns, we performed numerical nonlinear and multiphysics simulations to investigate the influence of the spatial distribution of MNPs in the scaffold on the quality of the hyperthermia treatment of bone tumors.

\section{MAGnetic SCAFFoldS PREPARATION}

\section{A. Magnetic Nanoparticles Synthesis and Characterization}

The preparation of iron oxide magnetic nanocrystals samples (MNC1 and MNC2) was performed through a modified partial oxidation route protocol [52]. Briefly, iron (II) sulfate $\left(\mathrm{FeSO}_{4} \bullet 7 \mathrm{H}_{2} \mathrm{O}\right)$ was let react under $\mathrm{N}_{2}$ atmosphere at $90^{\circ} \mathrm{C}$ with potassium nitrate $\left(\mathrm{KNO}_{3}\right)$ and potassium hydroxide ( $\mathrm{KOH}$, all from Sigma Aldrich) in water. MNCs were isolated from the reaction mixture by magnetic separation, washed and re-dispersed in water.

Samples features were investigated by transmission electron microscopy and X-ray diffraction (XRD). XRD patterns were recorded using $\mathrm{Cu}-\mathrm{K} \alpha$ radiation on a Panalytical Empyrean diffractometer equipped with a graphite monochromator on the diffracted beam and an X'Celerator linear detector.

Transmission electron microscopy (TEM) images were recorded on a Hitachi H-7000 instrument running at $125 \mathrm{kV}$ and on a Jeol JEM 1400 Plus operating at $120 \mathrm{kV}$. Samples for TEM observation were deposited on a C-coated copper grid. The XRD patterns (see Figure S1, in SM1 of the Supplementary Material) are quite similar for the two MNCs samples and are consistent with the formation of

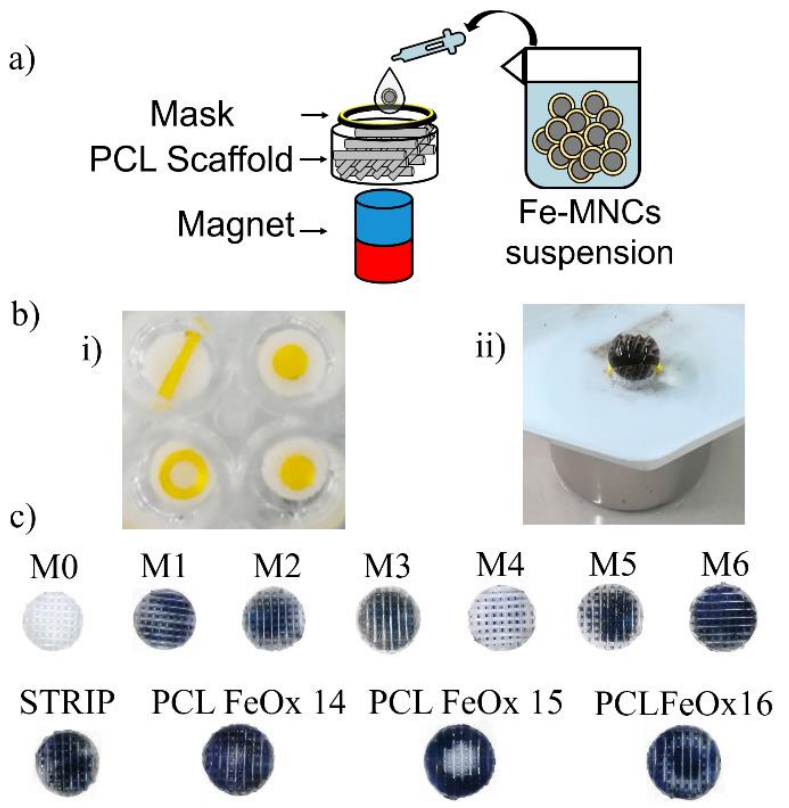

Fig. 3. a) Magnetic scaffolds fabrication: magnetic nanocrystals (MNCs), dispersed in water, are dripped onto the PCL scaffolds and the deposition is carried out under the driving force of the external magnet. b) i) Examples of arrangements of waterproof adhesive tape as masks for patterning the MNCs in the biomaterial ii) Example of the sample preparation during the drop-casting. c). Optical images of the PCL scaffolds loaded with magnetic nanocrystals as obtained by the proposed modified drop-casting process. The manufacturing method is controlled to result in a wide variety of magnetic nanoparticles distribution, and patterns in the biomaterial.

nanocrystalline iron oxide with a spinel structure which can be ascribed to the formation of magnetite $\left(\mathrm{Fe}_{3} \mathrm{O}_{4}\right)$ and/or maghemite $\left(\gamma-\mathrm{Fe}_{2} \mathrm{O}_{3}\right)$. The XRD peak broadening suggests that MNC1 features larger average crystalline domains as compared to MNC2. In particular, the average size of crystalline domains ( $\left.\langle d\rangle_{\mathrm{XRD}}\right)$ as assessed through the Scherrer equation based on profile fitting by Panalytical Highscore software and corrected by instrumental broadening using a lab reference, turned out to be $58 \mathrm{~nm}$ and $32 \mathrm{~nm}$ for MNC1 and MNC2, respectively.

TEM analysis supports the nanocrystalline nature of the two samples, indicating the occurrence of nanocrystals with cubic and cubo-octahedral morphology (see Figure S1), together with some elongated nanocrystals which appear in MNC1, likely associated with goethite. The average size of the nanocrystals is consistent with XRD data; in addition, TEM indicates that $\mathrm{MNC1}$ shows a broader size distribution as compared to MNC2.

\section{B. Scaffold Loading by Drop-Casting}

In this study, the polymeric scaffolds produced by 3D Biotek (3D Biotek LLC, NJ, USA) were purchased from Sigma-Aldrich (Merck KGaA, Darmstadt, Ge). These standard PCL disks have a $5 \mathrm{~mm}$ diameter and are $1.6 \mathrm{~mm}$ in height. The set of 3D-Insert is constituted by a $90^{\circ}$-interlaced fiber architecture, with a fiber diameter of $300 \mu \mathrm{m}$, spaced by 300 $\mu \mathrm{m}$, resulting in a nominal porosity of about $80 \%$. A representative image of the bare, unloaded PCL scaffold is reported as M0 in Fig. 3. 
In order to produce the MagS, the water-based dispersion of the MNCs was first sonicated and then a drop was deposited on the PCL scaffolds, as depicted in Fig. 3.a. NdFeB permanent magnet (Webcraft GmbH, DEU, S-20-20-N, diameter $10 \mathrm{~mm}$, height $20 \mathrm{~mm}, 20 \mathrm{~mm}, 4.5 \mathrm{~kg}$ of attraction force, $\sim 1 \mathrm{~T}$ at the surface) was located at the bottom of the PCL scaffold in order to promote the MNCs uptake. Finally, the scaffold was dried in a furnace at $40^{\circ} \mathrm{C}$, under static air.

For the production of magnetic scaffolds M1 to M6, sample MNC1 was used, and a ten magnet-guided deposition followed by drying was performed to achieve a different loading of nanoparticles. Scaffolds labeled as Strip, PCL FeOx 14-16 were prepared by deposition of sample MNC2. In this case, before the deposition process, a water-repellant tape with the desired pattern was applied to one side of the original PCL scaffold. The tape limits MNC deposition in the selected area, leading to non-homogeneous distribution of MNCs in the scaffolds, as shown in Fig. 3.b. Then, the tape was removed, and the scaffold was dried at $40^{\circ} \mathrm{C}$. The resulting MagS samples are shown in Fig. 3.c.

\section{METHODS FOR MAGNETIC SCAFFOLD CHARACTERIZATION}

\section{A. Static Magnetic Measurements}

Static magnetization measurements were carried out using the magnetic properties measurement system (MPMS XL 5, Quantum Design), applying a magnetic flux density up to a maximum of $7 \mathrm{~T}$, with a constant temperature of $310 \mathrm{~K}$ $\left(37^{\circ} \mathrm{C}\right)$, in order to replicate the susceptibility of the particles in physiological conditions.

The measured magnetization curves $(M$, in emu) were fitted to the following equation [53]:

$$
M(B)=\widetilde{\phi}_{m} M_{S, M N C}\left[\operatorname{coth}\left(\frac{\mu_{\mathrm{mnp}} B}{k_{B} T}\right)-\frac{k_{B} T}{\mu_{\mathrm{mnp}} B}\right]
$$

where $\widetilde{\phi}_{m}$ is the average amount of MNPs loaded in the biomaterial matrix, $M_{S, M N C}$ is the saturation magnetization of the single nanoparticle, $\mu_{m n p}$ is the nanoparticle longitudinal magnetic moment and $B$ is the applied external, longitudinal magnetic flux density (in T), $k_{B}$ is the Boltzmann's constant and $T$ is the system temperature (in $\mathrm{K}$ ). The fitting is performed using Matlab 2021a (The MathWorks Inc., MA, USA) considering as unknown the term $\widetilde{\phi}_{m}$.

The response of the manufactured magnetic scaffolds was also evaluated measuring the maximum distance of attraction in presence of a static magnetic field generated by the same permanent $\mathrm{NdFeB}$ magnet used during the drop-casting procedure.

\section{B. Morphological Characterization and Microstructure Observation}

The presence, clustering, and distribution of the MNPs in the polymeric matrix, as well as the scaffold internal morphology were verified with Scanning Electron Microscopy (SEM) imaging. The JEOL JSM-7500FA (JEOL, Jap) microscope from Electron Microscopy Facility at IIT (Genoa, IT) was employed to assess the spatial distribution of the MNCs in the produced magnetic scaffolds samples. The SEM images were analyzed using the open-source software Image Processing and Analysis in Java (ImageJ, NIH Gov.). The distributions of the pore size, the radii of the magnetic nanoparticles and the presence of clusters in the scaffolds were assessed.

\section{Differential Scanning Calorimetry and Thermogravimetric Analysis}

The simultaneous Differential Scanning Calorimeter (DSC)Thermogravimetric (TGA) analyzer SDT Q600 (TA, New Castle, DE) was used to determine the MNCs loading in a selected scaffold [40], [54], [55] A $40 \mu$ l alumina pan was used as a sample holder. The initial temperature was $20^{\circ} \mathrm{C}$. The protocol consists of a heating ramp with a slope of $20^{\circ} \mathrm{C} \cdot \mathrm{min}^{-1}$ up to the final temperature of $1000^{\circ} \mathrm{C}$, under $\mathrm{N}_{2}$ sample purge flow at a flow rate of $100 \mathrm{~mL} / \mathrm{min}$.

The temperature values at different mass percentages (T10\% T50\% T90\%) are considered. The first and second derivatives of the weight vs. Temperature were investigated.

\section{THz Tomography}

Nowadays, several approaches are considered for characterizing the pore size, distribution, and architecture of biomaterials [56], [57], as well as drug loading capabilities or functional properties [58], [59]. Among these, THz imaging is of interest because it allows for sample characterization without compromising its integrity. Herein, THz Time of flight (TOF) imaging technique [60] has been exploited to estimate the spatial distribution of MNPs in the PCL scaffolds.

$\mathrm{THz}$ TOF Imaging, also known as $\mathrm{THz}$ pulsed imaging (TPI), has the unique property of providing a 3D "map" of the object by exploiting data collected in reflection mode [60].

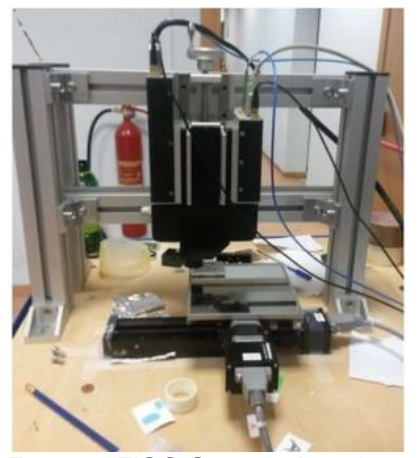

Fig. 4. Zomega FiCO System: Imaging module

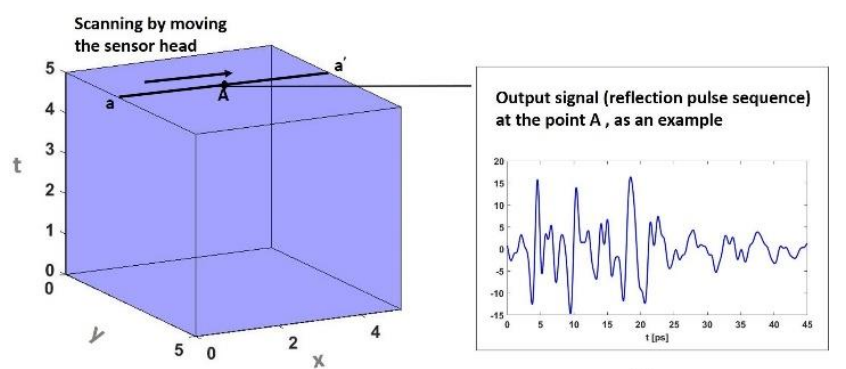

(a)

(b)

Fig. 5. Data Measured by the FiCO system. (a) Acquisition of THz data along a line a-a'. (b) Single point measurement. 
In brief, the object is probed by a pulse signal and the reflected waveform is collected as a time-dependent function in a certain observation time window. The reflected pulses and their temporal delay reveal the internal structure of the sample (if the object is nonmetallic). It is, indeed, worth pointing out that the time of flight (TOF), i.e., the time $t$ that the waveform employs to propagate from the emitter to an electromagnetic discontinuity and to go back to the receiver, is related to the distance $d$ between $\mathrm{THz}$ probes and the detected discontinuities as

$$
t=2 \cdot \frac{d}{v}
$$

$v$ being the electromagnetic wave propagation velocity into the object.

$\mathrm{THz}$ data have been collected by means of the Zomega $\mathrm{THz}$ FiCO system [61]-[63] available at the Institute for Electromagnetic Sensing of the Environment - National Research Council of Italy (IREA-CNR). The system is equipped with an ad hoc designed imaging module (see Fig. 4) and collects data in normal reflection mode in $40 \mathrm{GHz}$ up to 3 $\mathrm{THz}$ frequency range. Fig. 5 describes the measurement process of the FiCO system. The system provides 3D data (2D in space $+1 \mathrm{D}$ in time) collected using an automatic planar scan, moving along $\mathrm{x}$ and $\mathrm{y}$ directions (see Fig. 5.a), within a 100 ps observation time window. At each measurement point, the air-object interface as well as all the electromagnetic discontinuities inside the object generate reflections, which are recorded as a time-dependent waveform. Fig. 5.b shows an example of a single-point measurement.

$\mathrm{THz}$ data have been gathered on a $10 \mathrm{~mm} \times 10 \mathrm{~mm}$ wide scan area, which is discretized by square pixels whose side is $0.12 \mathrm{~mm}$, for all the samples analyzed (see Fig. 3.c). The samples have been placed on a support to have a unique spatial reference.

$\mathrm{THz}$ data have been processed by means of the procedure detailed in Appendix, which provides two main outputs:

- The Magnetization Index $\left(I_{m}\right)$, which encodes the amount of MNPs present in the sample under test. The $I_{m}$ is calculated from the $2 \mathrm{D}$ binarized magnetization map (Fig. 13h in Appendix) as the ratio between the number of pixels containing MNPs (yellow pixels marked with 1 in Fig. 13h) and the total number of pixels discretizing the scanning area and intercepting the sample.

- The 2D MNPs distribution map (Fig. 13i) shows the parts of the sample under test where MNPs are mostly concentrated and allows to appraise the potential of the manufactured magnetic scaffolds for the hyperthermia treatment of bone tumors. This map is obtained by multiplying pixel by pixel the binary magnetization map times (Fig. 13h) and the map of the filtered $\mathrm{THz}$ signal amplitude (Fig. 13b).

\section{E. In Silico Tests of Hyperthermia Treatment with Magnetic Scaffolds}

The aim of this work is to investigate if and how the loading patterns of magnetic scaffolds could affect the hyperthermia treatment of residual bone tumor cells, shown in Fig. 1. We

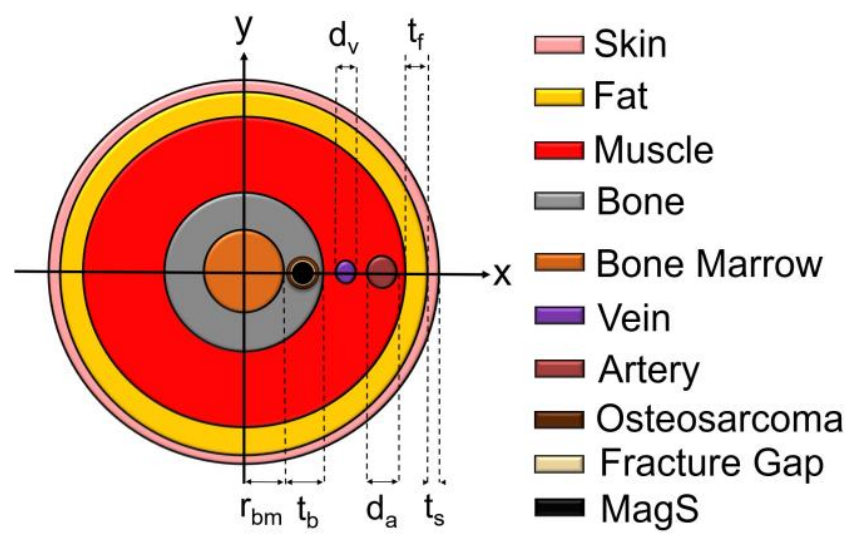

Fig. 6. Geometry for the in silico study of hyperthermia treatment of bone tumors with magnetic scaffolds (MagS). The system geometry is a layered model of a physiological arm with a surgically reduced osteosarcoma tumor, having radius $r_{t}=5 \mathrm{~mm}$. A MagS, with radius $r_{s c}$ $=5 \mathrm{~mm}$, is assumed to be implanted for bone repair.

characterized the physiochemical properties of MagS implemented through the drop-casting and derived essential information for performing extensive and accurate in silico experiments of the hyperthermia treatment of bone tumors (as shown in Fig. 2).

As depicted in Fig. 9, a MagS, with a radius of $5 \mathrm{~mm}$, is assumed to be implanted after the surgical resection of an osteosarcoma tumor, which leaves a margin of residual cells $0.5 \mathrm{~mm}$ thick, grown between the marrow and the cortical region of a human upper limb [48]. The system geometry, shown in Fig. 6, is a layered surface phantom consisting of a skin layer with thickness $t_{s}$ of $1.5 \mathrm{~mm}$, a fat and a muscle layer having thicknesses $t_{f}=10 \mathrm{~mm}$ and $t_{m}=36 \mathrm{~mm}$, respectively. The bone humerus, with radius $r_{b m}+t_{b}=20 \mathrm{~mm}$ and the bone marrow, having radius $r_{b m}=10 \mathrm{~mm}$, are taken as the center of the coordinates system. The brachial artery and vein were considered in the geometry to account for the heat transfer [48]. The system is assumed to be exposed to the magnetic field generated from a solenoid working at frequency $f$. The electromagnetic properties of the tissues are taken from [48] and reported in the Supplementary Material.

In this framework, the spatiotemporal dynamics of the temperature of the biological tissues during the HT can be studied by solving the Pennes' Bio-Heat equation [48]:

$$
\rho C_{p} \frac{\partial T}{\partial t}=\nabla \cdot(k \nabla T)-\rho_{b} \omega_{b} C_{p, b}\left(T-T_{b}\right)+Q_{E M}
$$

where $\rho$ is the tissue density in $\mathrm{kg} \cdot \mathrm{m}^{-3}, C_{p}$ is the specific heat capacity in $\mathrm{Jkg}^{-1} \mathrm{~K}^{-1}, k$ is the thermal conductivity in $\mathrm{Wm}^{-2} \mathrm{~K}^{-1}$. The terms $\rho_{b}, C_{p, b}$ and $\omega_{b}$ indicates the blood thermal properties and blood perfusion (in $\mathrm{s}^{-1}$ ), respectively. The thermal properties are reported in sect. SM2 of the Supplementary Material.

Eq. (3) is solved assuming as initial distribution the steadystate temperature distribution resulting in the equilibrium of a $37^{\circ} \mathrm{C}$ for all tissues and an air temperature of $25^{\circ} \mathrm{C}$. Heat exchange by convection is assumed at the skin-air interface, given a convection heat transfer coefficient of $7.7 \mathrm{Wm}^{-2} \mathrm{~K}^{-1}$. At the muscle-vessel boundaries an effective convection 
mechanism is assumed [48].

The total electromagnetic (EM) power per volume unit dissipated during the HT in the system is $Q_{E M}$, which is the sum of the power dissipated by the induced RF currents $\left(P_{e}=\right.$ $\sigma|E|^{2}$, where $\sigma$ is the electrical conductivity, in $\mathrm{Sm}^{-1}$, and $E$ is the electric field in $\mathrm{Vm}^{-1}$ ) and the magnetic losses due to the implanted MagS, i.e. [48]:

$$
P_{m}=\pi f \mu_{0}|\boldsymbol{H}|^{2} \chi^{\prime \prime}
$$

where $f$ is the working frequency, in $\mathrm{Hz}, \mu_{0}$ is the vacuum magnetic permeability equal to $4 \pi \cdot 10^{-7} \mathrm{Hm}^{-1}, \boldsymbol{H}$ is the magnetic field in $\mathrm{Am}^{-1}$, whilst $\chi^{\prime \prime}$ is the imaginary, out-ofphase part of the magnetic susceptibility of the thermo-seed. The external magnetic field is turned on at $\mathrm{t}=1 \mathrm{~min}$ and off at $\mathrm{t}=80 \mathrm{~min}$.

For populations of MNCs in solids or highly viscous biomaterials matrix, constrained to a noticeable particleparticle level of interactions, the complex magnetic susceptibility spectra $\left(\chi(f)=\chi^{\prime}-j \chi^{\prime \prime}\right)$ follows of a Cole-Cole model [48], [53], [64]:

$$
\chi(f)=\frac{\chi_{0}}{1+\left(2 j \pi f \tau_{N}\right)^{1-y}}
$$

where $\chi_{0}$ is the equilibrium susceptibility, $\tau_{N}$ is the characteristic Néel relaxation time, in $\mathrm{s}$, which accounts for a single dipolar relaxation mechanism and $\gamma$ is the broadening parameter, set equal to 0.75 [53].

For a group of cubic nanoparticles having a volume $V_{m}$ $\left(\mathrm{m}^{3}\right)$, with a $M_{s}\left(\mathrm{Am}^{-1}\right)$, the static magnetic susceptibility is defined as:

$$
\chi_{0}=\frac{\mu_{0} \phi_{m} V_{m} M_{S, M N C}^{2}}{k_{B} T}
$$

where $k_{B}$ is the Boltzmann' constant. In Eq. (6), in a way different from other works, the term $\phi_{m}$, i.e., the amount of nanoparticles inside the scaffold is assumed to be an unknown function of space:

$$
\phi_{m}=\phi(x, y)
$$

The loading pattern $\phi(x, y)$, which depends on the processing conditions, is obtained from $\mathrm{THz}$ tomography, as shown in Fig. 2. Fig. 6 and Fig. 7. We used the post-processed THz images to derive a closed-form equation for each of the ten scaffolds shown in Fig. 3.c. To this aim, the MNCs distributions were translated and fitted to a finite series of radial basis functions using Matlab "Isqcurvefit" function (see section SM3 in the Supplementary Material).

Previous literature works [48], [53], [64], considered $\widetilde{\phi}_{m}$, i.e., the volume-averaged amount of MNPs in the biomaterial, in Eq. (6), which can be derived from the static magnetic measurements or DSC-TGA analysis. However, this assumption leads to a misleading estimation of the hyperthermia treatment parameters [48].

The power dissipated by the MagS [Eq. (4)] depends linearly on the particle volume and quadratically from the saturation magnetization of the MNCs, but it also depends linearly on the volume fraction. It is worth noting that the wavelength of the RF field applied for performing the interstitial HT is much higher than the size of the implant and the average spatial variation of the MNPs profile (i.e., $\lambda_{t} \gg d \phi(x, y) / d r$ - few meters compared to sub-mm features). Despite the sub-wavelength variation, the EM problem can be approximated to a quasi-static regime and, from the thermal point of view, significant differences in terms of final tumor temperature and treatment quality were already demonstrated numerically [48].

The Néel relaxation time is a non-linear function of temperature [48]:

$$
\tau_{N}=\tau_{0}(T) e^{\frac{K_{A} V_{m}}{k_{B} T}}
$$

where $\tau_{0}(T)$ is a pre-exponential, precession factor defined as in [48] and $K_{A}$ is the crystal anisotropy energy in $\mathrm{Jm}^{-3}$.

The HT of bone tumors with MagS is a non-linear and multiphysics problem [48], [64]. Indeed, dissipated power from tissues and scaffolds is not constant during the treatment, but changes as the system temperature modify. The dielectric permittivity and electrical conductivity of tissues, $\sigma$, is assumed to vary with temperature (see SM2) [48]. Furthermore, the thermal properties cannot be considered as constant, especially the blood perfusion of healthy and tumor tissues [48]. Therefore, the coupled electromagnetic-thermal problem was solved using a frequency-transient solver with Comsol Multiphysics v5.5 (Comsol Inc., Burlinghton, MA USA) [48].

\section{Results}

\section{A. Characterization}

The static magnetic response of the magnetic scaffolds manufactured with the proposed drop-casting technique was investigated. The saturation magnetization values for the used MNCs, at room temperature, are expected to be superparamagnetic and in the range from 70-80 $\mathrm{Am}^{2} \mathrm{~kg}^{-1}$, based on investigation of similar samples [53]. The static magnetic response of the MagS manufactured by the dropcasting procedure is presented in Fig. 7.a. All the scaffolds present a superparamagnetic behavior, with negligible coercive forces. The MagS with the higher saturation magnetization are the sample M6 and PCLFeOx16 (Fig. 3.c), as can be observed by Fig. 7.a ( $\sim 8.8$ and $7 \mathrm{emu} / \mathrm{g})$. This strong magnetic characteristic is due to the $12.1 \%$ of MNCs loaded in it. From Tab. I, despite these two exceptions, it is possible to notice that samples loaded with similar MNCs populations present a coherent distribution of saturation magnetization and loading values (i.e., M1-M5 scaffolds, loaded with MNC1 particles, present very similar saturation magnetization and loading values). From the findings reported in Fig. 7.a and Tab. I, it is possible to notice that the drop-casted method for manufacturing MagS results in average saturation magnetization of about $3.062 \pm 2.664 \mathrm{emu} / \mathrm{g}$, for an average loading of about $2.420 \%$. The static magnetic measurements are confirmed by the attraction distances shown in Fig. 7.b. Indeed, for the M6 and PCLFeOx16, the measured minimum 
attraction distance of $2.5-3 \mathrm{~cm} \pm 0.2 \mathrm{~cm}$.

The results of TGA characterization for the bare (M0) and MNCs-loaded scaffold (M5) are reported in Fig. 8. The TGA curve of the bare PCL scaffold (M0) shows a single relevant weight loss which takes place at around $400^{\circ} \mathrm{C}$. The thermal behavior of the polymeric matrix is coherent to the analysis from [54], [55]. This main mechanism of weight loss is also observed in the MNCs-loaded (M5) scaffold. On the other hand, as no mass variations due to the PCL substrate are expected above this temperature, the weight at $600^{\circ} \mathrm{C}$ was used to assess the MNCs overall loading in the substrate $\left(\widetilde{\phi}_{m}\right)$, which in the case of the M5 scaffold turned out to be $\sim 1.89$ wt. $\%$, for an approximate weight of $0.036 \mathrm{mg}$ of magnetite.

The value of MNCs loaded in the scaffold found by DSCTGA is very similar to the values obtained by the fitting procedure, resulting in a difference below $1 \%$ (Tab. I).

The MNCs distributions for the ten MagS are reported in Fig. 9. From the processing of the high-THz signals and scans (Sect. III.B), the refractive index $(n)$ and the magnetization index $\left(I_{m}\right)$ were derived and reported in Tab. I. These physical quantities were investigated as a function of and correlated to the average volume fraction of MNCs in the scaffolds and the saturation magnetization, as shown in Fig. 10. From Fig. 10, it is possible to notice that both
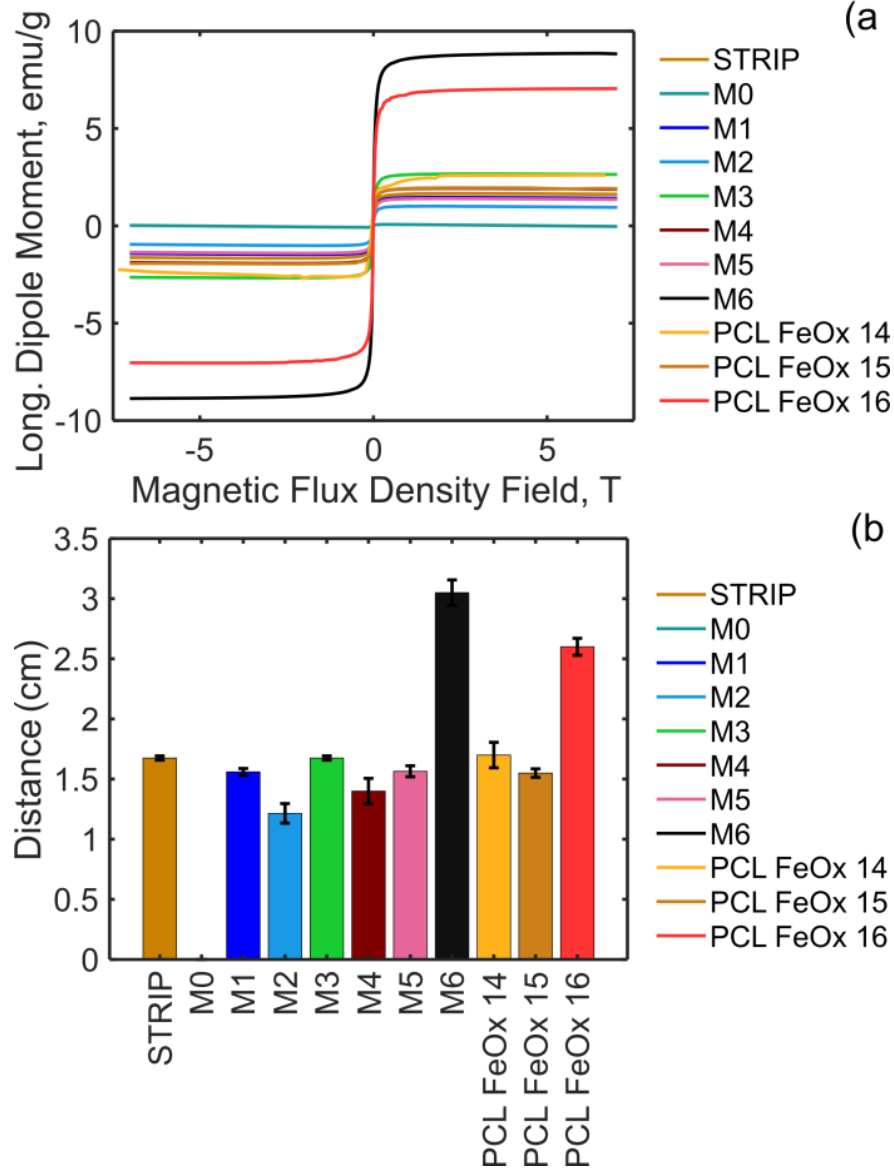

Fig. 7. a) Scaffold magnetization as a function of an external static magnetic field for the ten scaffolds (M1-M6, PCL FeOX 14 - PCL FeOx 16) and the reference, unloaded PCL disk (M0). b) Minimum attraction distance $(\mathrm{cm})$ from the $\mathrm{NdFeB}$ magnet.

TABLE I
MAGNETIC MEASUREMENT FITTING AND PROCESSING OF THz

\begin{tabular}{ccccccc}
\multicolumn{7}{c}{ SCANS } \\
Sample & $\begin{array}{c}\mathrm{Ms} \\
\left(\mathrm{emu} \cdot \mathrm{g}^{-1}\right)\end{array}$ & $\widetilde{\phi}_{m}(\%)$ & $\mathrm{R}_{2}$ & $\begin{array}{c}\mathrm{d} \\
(\mathrm{mm})\end{array}$ & $\mathrm{n}$ & $\mathrm{I}_{\mathrm{m}}$ \\
\hline M0 & 0.0001 & 0 & - & 1.30 & 1.73 & 0 \\
M1 & 1.4672 & 1.9987 & 0.9867 & 1.28 & 1.80 & 0.46 \\
M2 & 1.0103 & 1.3610 & 0.9991 & 1.27 & 1.75 & 0.30 \\
M3 & 2.6723 & 3.6629 & 0.9894 & 1.4 & 1.76 & 0.50 \\
M4 & 1.9269 & 2.6322 & 0.9654 & 1.5 & 1.74 & 0.14 \\
M5 & 1.400 & 1.9059 & 0.9904 & 1.36 & 1.81 & 0.46 \\
M6 & 8.8664 & 12.1011 & 0.9231 & 1.20 & 1.84 & 0.66 \\
PCL FeOx 14 & 2.6118 & 2.0142 & 0.9663 & 1.26 & 1.82 & 0.80 \\
PCL FeOx 15 & 1.9609 & 2.387 & 0.9815 & 1.21 & 1.81 & 0.49 \\
PCL FeOx 16 & 7.0472 & 8.466 & 0.9270 & 1.24 & 1.80 & 0.34 \\
STRIP & 1.6642 & 2.026 & 0.9670 & 1.30 & 1.79 & 0.45 \\
\hline
\end{tabular}

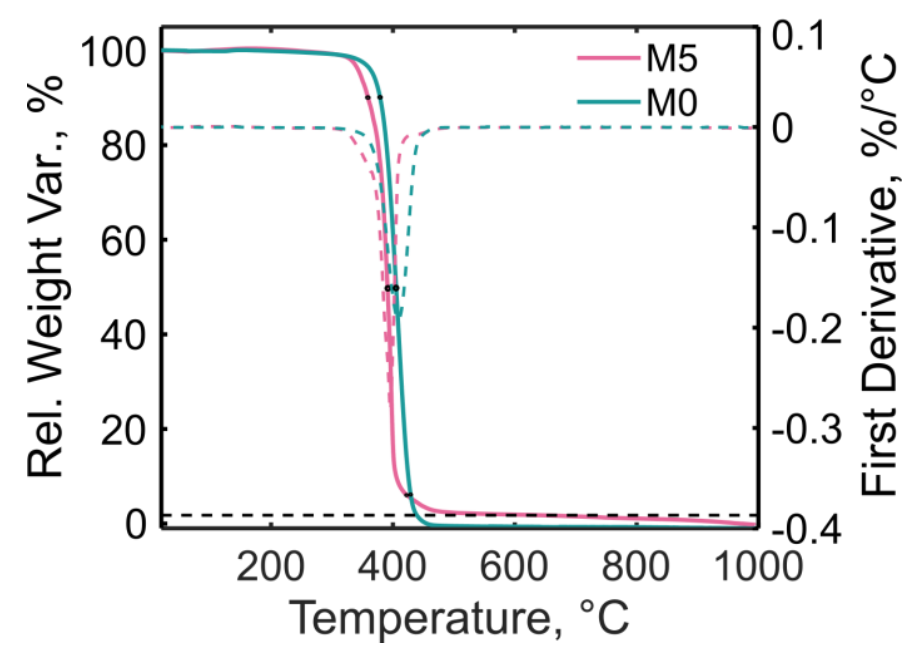

Fig. 8. Example of the relative weight $\left(\mathrm{W}_{\%}\right)$, and its first derivative, versus temperature for the sample M5. The temperatures at $90 \%, 50 \%$ and $10 \%$ of the initial weight are highlighted. The residual magnetite mass is evaluated at $600^{\circ} \mathrm{C}$.

indexes increase in a non-linear, sigmoid-like fashion for increasing content of $\mathrm{MNCs}$ and saturation magnetization values. The average refractive index is about $1.785 \pm 0.35$, with a maximum difference of $3.5 \%$ between the highly magnetized M6 sample and the reference, unloaded PCL matrix. In this framework, the proposed magnetization index $\left(I_{m}\right)$ allows coping with smaller differences between the different MagS (Tab. I, Fig. 10).

\section{B. Simulations Results}

The samples manufactured by the drop-casting procedure, shown in Fig. 3, were characterized by SQUID magnetometry to obtain the scaffold saturation magnetization $\left(M_{s}\right)$, the maximum amount of MNPs in the sample $\left(\tilde{\phi}_{m}\right)$ and the loading pattern of the biomaterial $(\phi(x, y))$. As depicted in Fig. 2, these information were used to simulate the hyperthermia treatment of bone tumors using the different MagS samples. By using the profiles derived from $\mathrm{THz}$ scans, shown in Fig. 8, the hyperthermia treatment of MagS was investigated in silico.

Given the fitting parameters from Tab. I, the magnetic power losses $\left(P_{m}\right.$, Eq. (4)) for MagS were estimated and the distributions are shown in Fig. 11.a-11.c. In the simulations of the hyperthermia treatment of bone tumors we set the 


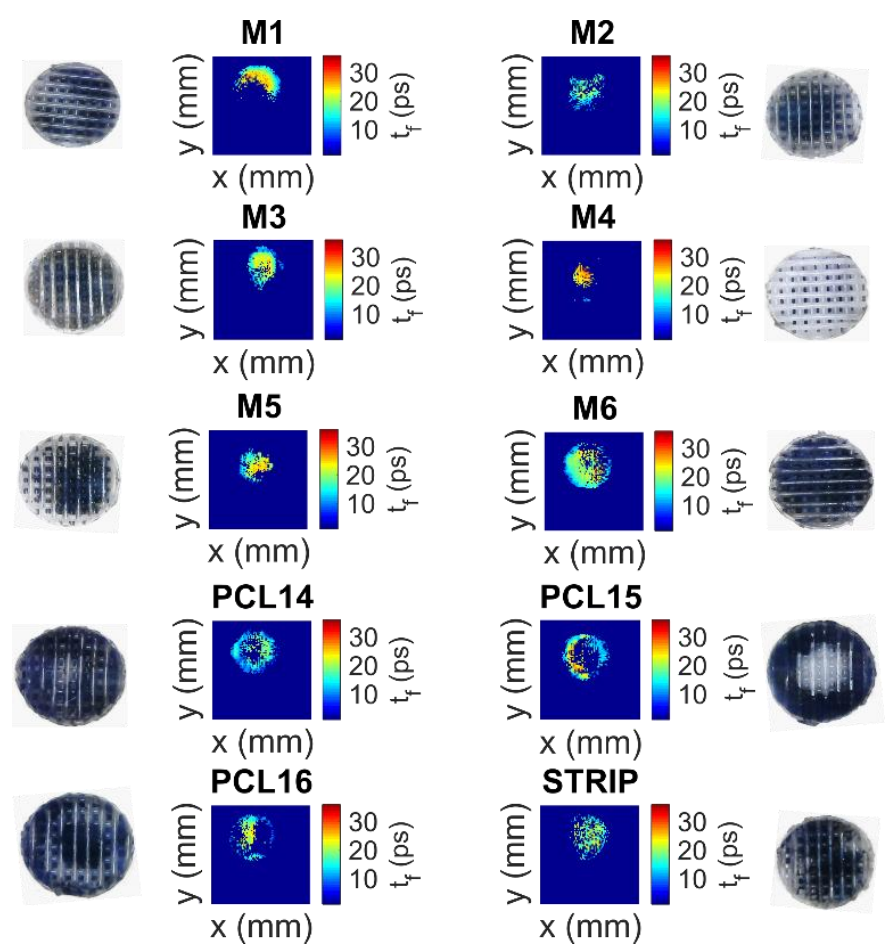

Fig. 9.: The 2D MNCs distribution maps for the eight magnetic scaffolds manufactured with drop-casting method. The aggregated results in terms of Magnetization indexes $\left(I_{m}\right)$ are reported in Tab. I.

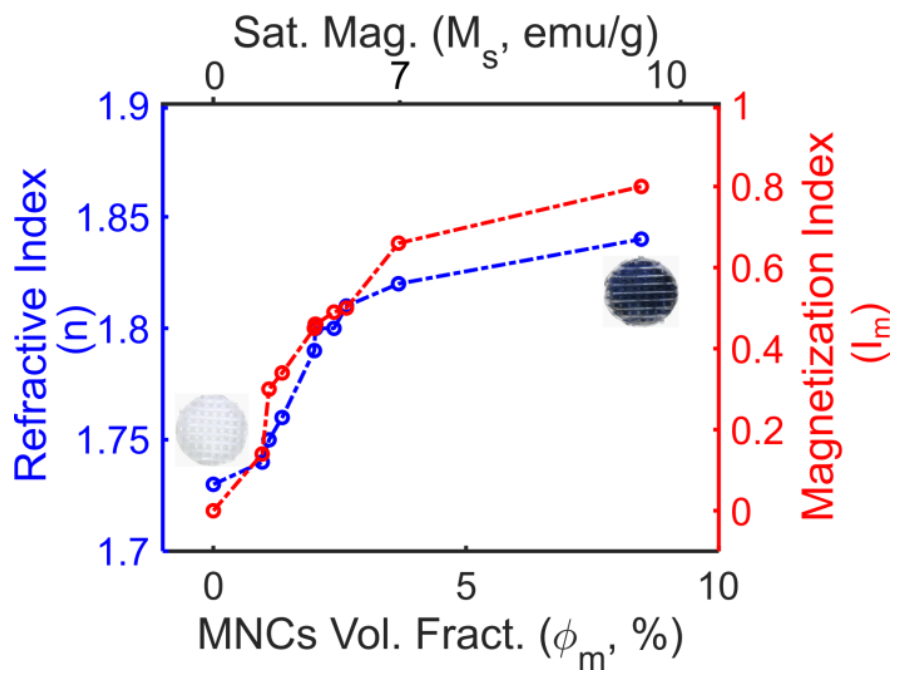

Fig. 10. Refractive index (n) and magnetization index (Im) as a function of the loaded volume fraction of magnetic nanocrystals (MNCs), $\widetilde{\phi}_{m}$, and of the saturation magnetization of the magnetic scaffolds (in emu/g). The unloaded PCL, reference scaffold M0 and the sample M6 are shown.

magnetic field to an amplitude of $30 \mathrm{mT}$ and a working frequency of $300 \mathrm{kHz}$. From Fig. 11.a-.c is possible to notice that MagS loaded with MNC2 exhibit a higher hyperthermia potential. Indeed, the power per volume unit dissipated is about one order of magnitude higher than those of MNC1loaded scaffolds (Fig. 11.a-.b). This is due to the smaller size of the MNC2 nanoparticles (32 nm vs. $58 \mathrm{~nm}$ ) [68]. The effect of loading amount on the final dissipated power was also investigated (Fig. 10.c). The results based on two selected loading values show similar trends with temperature.
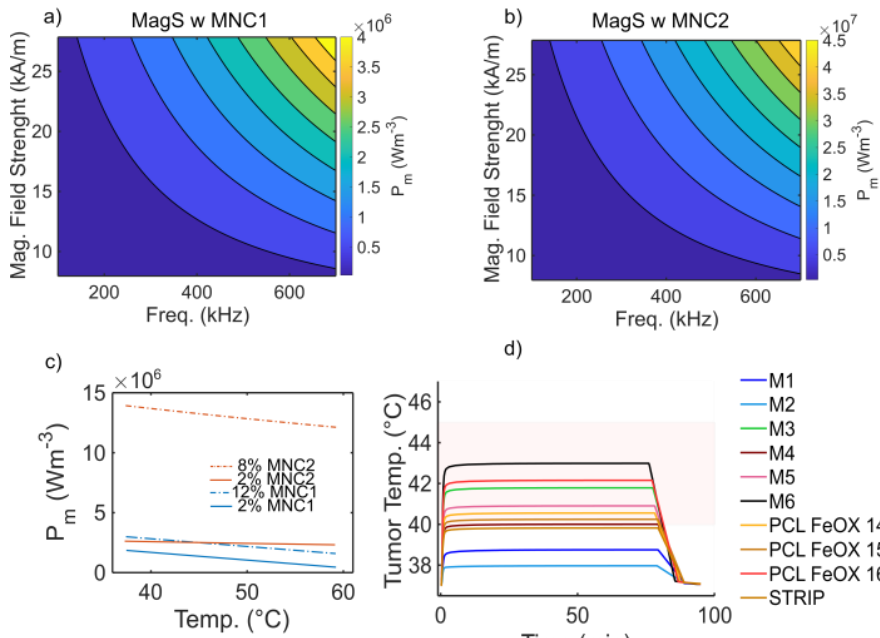

d)

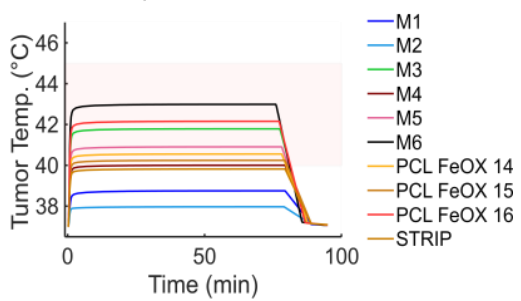

e)

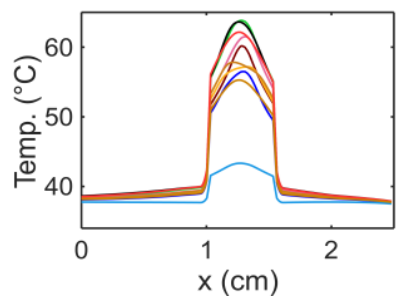

f)

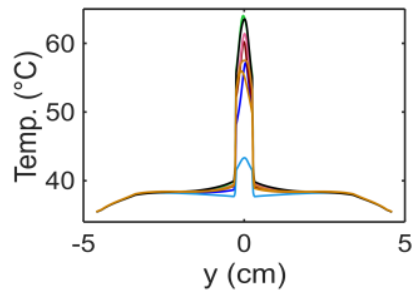

Fig. 11. a) Power per volume unit (Pm, Wm-3) dissipated by a dropcasted MagS with $8 \%$ of MNC1, for different magnetic field strength and working frequencies. b) Power per volume unit $\left(\mathrm{Pm}, \mathrm{Wm}^{-3}\right)$ dissipated by a drop-casted MagS with $8 \%$ of MNC2 for different magnetic field strength and working. c) Power per volume unit (Pm, $\mathrm{Wm}^{-3}$ ) dissipated by drop-casted scaffolds made with MNC1 and MNC2 for average and maximum values of the volume fraction, evaluated as a function of temperature, at $H=30 \mathrm{mT}$ and $f=300 \mathrm{kHz}$. d) T50 vs. time for the drop-casted magnetic scaffolds. The shaded area represents the therapeutic range. e) Temperature profile along the $x$-coordinate $(y=0)$, at $t=80 \mathrm{~min}$, for all the magnetic scaffolds. e) Temperature profile along the $y$-coordinate, passing through the scaffold, at $t=80 \mathrm{~min}$, for all the magnetic scaffolds.

Multiphysics, non-linear simulations were used to investigate how the spatial distribution could also influence the power deposited power, and are shown in Fig. 11.d-.f. From the analysis of the time evolution of the average temperature in the osteosarcoma tumor region (Fig. 11.d), the scaffolds M3, M5 and M6, but also PCLFeOx14-16 can reach and withstand the therapeutic temperature range, whilst the features of M1, M2 do not enable effective treatment. Under the simulated treatment conditions, the samples M4 and STRIP are very close to the threshold value and a slight adjustment to the extrinsic field parameters would result in an enhanced dissipation. The very different therapeutic outcome could be understood by observing that temperature patterns, at the final time of $80 \mathrm{~min}$, along the $x$ - and $y$-directions varies for all the magnetic scaffolds differs, depending on the loading pattern, in terms of peak height symmetry and broadness, as shown in Fig. 11.e and Fig. 11.f.

\section{DISCUSSION}

The results presented in Tab. I and Fig.s 7-11 elucidate that the loading patterns of magnetic scaffolds can affect the potential therapeutic capability of these multifunctional devices against bone cancers, regardless the features of the MNCs. 
A first explanation can be ascribed to the fact that the different spatial loading can impact the static magnetic properties of the sample, as shown in Fig. 7 and Tab. I. The drop-casting method developed for obtaining different loading pattern of magnetic scaffolds, can result in rather different distributions of the MNCs in the samples and, therefore, clusters can form, which may influence negatively the final magnetic properties after the loading procedure. In this framework, the possibility of non-linear magnetic effects due to the loading may arise [40], [43], [45], [66]. From the SEM images reported in Fig. S5 (Sect. SM4, Supplementary Material) it can be noticed that the MNCs are mostly distributed on the surface of PCL fibers, but the iron presence can be tracked also within the low magnetization samples M2 and M4. As confirmed by Fig. S6, the magnetization procedure causes some regions where the MNCs are concentrated and presenting large $(\sim 23 \mu \mathrm{m})$, irregular aggregates, which determines an increase in the particleparticle interaction level. The manufactured MagS presents appealing magnetic features, concerning the available literature counterparts. Indeed, the attraction distances shown in Fig. 7.b are comparable to those reported in [28] for cylindrical PGA scaffolds of $0.9-1.5 \mathrm{~cm}$ in diameter, with 2$8 \%$ loading of commercial $20 \mathrm{~nm}$ magnetite particles $\left(M_{s}\right.$ ranging from 2.5 to $8 \mathrm{emu} / \mathrm{g}$ ). The magnetization of our dropcasted MagS results in larger attraction distances, higher than the values of 0.4-0.8 cm reported in [55] for PCL scaffolds embedding $5 \%$ and $10 \%$ of $10 \mathrm{~nm}$ magnetite nanoparticles. These magnetic features make our drop-casted MagS appealing for bone tissue engineering [23]-[47], but especially for bone tumor hyperthermia [48].

Given the promising comparison with other magnetic scaffolds synthesized and characterized for bone tumor hyperthermia [17], [26], [37], [38], [42], we evaluated the hyperthermic potential of the drop-casted magnetic scaffolds in silico, for the geometry in Fig. 6 [48]. The extrinsic, treatment parameters of $30 \mathrm{mT}$ and $300 \mathrm{kHz}$ are a reasonable trade-off between heat administration, healthy tissue safety [3], [67], while being in accordance with other literature studies [38], [42], [48]. The fact the MNCs in the scaffold are not homogenously distributed, as shown in Fig. 9, can affect the final heating performances against the residual osteosarcoma cells. As figures of merit, we consider the spatial variation of the temperature along the $x$ - and $y$-axis and the T50, defined as the iso-temperature that cover at least 50\% of the tumor volume, and allows to predict standard hyperthermia treatment quality [51], [69]-[71]. From Fig. 11.d, it is possible to notice that M1, M2 and STRIP samples would not be capable of reaching the therapeutic range, despite their saturation magnetization and average loading fraction (Tab. I) were promising and comparable to the other samples. However, by considering the MNCs distributions retrieved from $\mathrm{THz}$ scans (Fig. 9), part of the scaffold volume is not occupied by the MNCs, as supported by the computed magnetization index. By investigating the spatial distribution of the temperature inside the MagS, shown in Fig. 11.e and 11.f, we can support this assumption by underlining that the temperature gradients for these samples are too narrow and, in an anisotropic fashion, the heat diffusion to the surrounding target residual cancerous cells is not homogeneous.
Furthermore, by observing Fig. 10.d, it is possible to infer that the set of MagS drop-casted with MNC1 particles in a relatively more homogenous way (Fig. 9), i.e., M3, M5 and M6 are promising candidates for performing the hyperthermia treatment of bone tumors. Indeed, after $80 \mathrm{~min}$ of treatment, these thermo-seeds could reach T50 values of $42 \pm 11.8^{\circ} \mathrm{C}$, $40.9 \pm 1.5^{\circ} \mathrm{C}$ and $43 \pm 1.75^{\circ} \mathrm{C}$, respectively. This is a noticeable result, since, from Fig. 11.c, a reduced hyperthermia potential was expected. However, taking into account the spatial influence of the MNCs distribution, shown in Fig. 11.e and 11.f, the broader spatial arrangement can ensure a significant therapeutic hyperthermia treatment. On the other hand, the samples PCL FeOx 15 and 16, which presented an annular distribution of magnetic particles with high power losses, are also a valuable solution for administering the heat to the residual osteosarcoma cells. Indeed, together with the huge void volume in the biomaterial and the observed low magnetization index (Tab. I) the differences in MNCs with different features and the temperature gradients which are kept steep lowering the distance from the target should be taken into account (Fig. 11.e, Fig. 11.f).

\section{CONCLUSIONS}

This work dealt with the investigation of the effects of the magnetic nanoparticles loading patterns on the hyperthermia potential of magnetic scaffolds against bone tumors. In particular, we developed a drop-casting method that allowed us to provide a set of different spatial arrangements of MNCs in a PCL matrix. Then, magnetic, calorimetricthermogravimetric and $\mathrm{THz}$ tomography characterization was carried out, combining the information for developing a unique multiphysics, non-linear model to investigate, in silico, the influence of the loading pattern on the quality of the hyperthermia treatment. From our analysis, we found that together with the intrinsic properties of the magnetic particles, their spatial arrangement in the biomaterial could be sought to perform an effective treatment. It should be pointed out, however, that the heating of the target volume can present a large deviation of the temperature distribution, i.e. an average standard deviation of $\pm 1.5^{\circ} \mathrm{C}$ around the therapeutic threshold values (Fig. S7, Supp. Mat).

Overall, our investigation can provide valuable feedback and state that the manufacturing of homogenously loaded magnetic scaffolds is advisable, although, the strategy of loading the outer edges of the scaffold can perform effectively. From our numerical study, to plan hyperthermia treatment with magnetic scaffolds, the loading and spatial distribution of MCNs must be controlled and reconstructed. In this work, we have demonstrated that this crucial information can be extracted using $\mathrm{THz}$ tomography. The proposed approaches and our findings could be relevant to material scientists, bioengineers and clinicians who aim to manufacture, characterize, and use magnetic scaffolds as multi-functional tools against, but not limited to, bone tumors. 


\section{APPENDIX}

\section{THZ DATA PROCESSING}

The ad-hoc procedure developed to process $\mathrm{THz}$ data is articulated in two main processing steps: i) Signal Filtering and ii) MNCs Distribution Estimate, see Fig. 12. In Fig. 13 output of each step along with the optical image of the sample PCL-FeOx15 are given.

The Signal Filtering step consists in the application of a band pass filter followed by a singular value decomposition (SVD) procedure (Fig. 12). The band pass filter is designed to select the effective spectrum of the signal [60] and is applied to the raw data. Then the SVD of the data matrix is computed and exploited as further filtering [61]. The aim is to reduce low and high-frequency noise affecting collected data. The false-color images in Fig. 13.a and Fig. 13.b show, at each investigated pixel, the maximum amplitude of the raw collected data and the filtered ones, respectively.

The MNCs Distribution Estimate aims at providing a 2D image representing a map of the MNCs distribution and consists of four steps as described in the flow chart shown in Fig. 12: 1) Refractive Index Estimation; 2) Propagation time delay map; 3) Threshold Estimate; 4) MNCs Distribution.

The Refractive Index Estimation aims to characterize the object under test from an electromagnetic point of view. In particular, $v$ is derived from the time-of-flight formula Eq. (2) in which $t$ is the time that the waveform needs to propagate inside the object and $\mathrm{d}$ is the thickness of the sample. The time-of-flight $t$ has been estimated from an A-Scan obtained by averaging the collected waveforms of the scanned area intercepting the sample as $t=t_{2}-t_{1}$ (see Fig. 14), where $t_{1}$ corresponds to the beginning of the scaffold (air/object interface) and $t_{2}$ corresponds to the temporal position of the last peak due to object/support interface. On the other hand, the thickness of the samples is a known value and is shown in Tab. I for all samples. The refractive index is given by $n=c / v$ being $\mathrm{c}$ the propagation velocity in vacuum. The results obtained for all the samples analyzed are shown in Tab. I.

The propagation time delay map is evaluated through the following procedure.

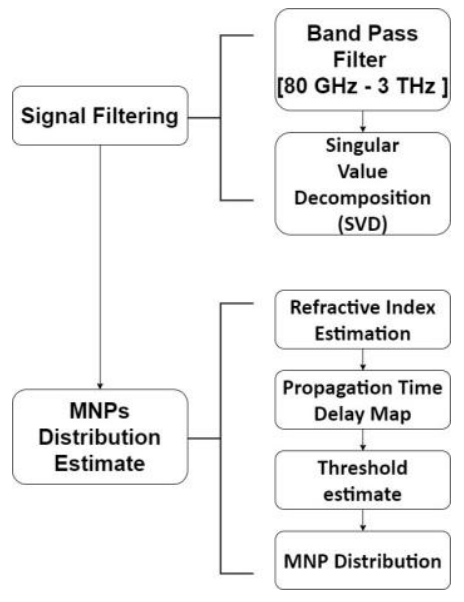

Fig. 12. Multi Steps procedure used to extract 2D magnetic nanoparticles (MNPs) distribution map.

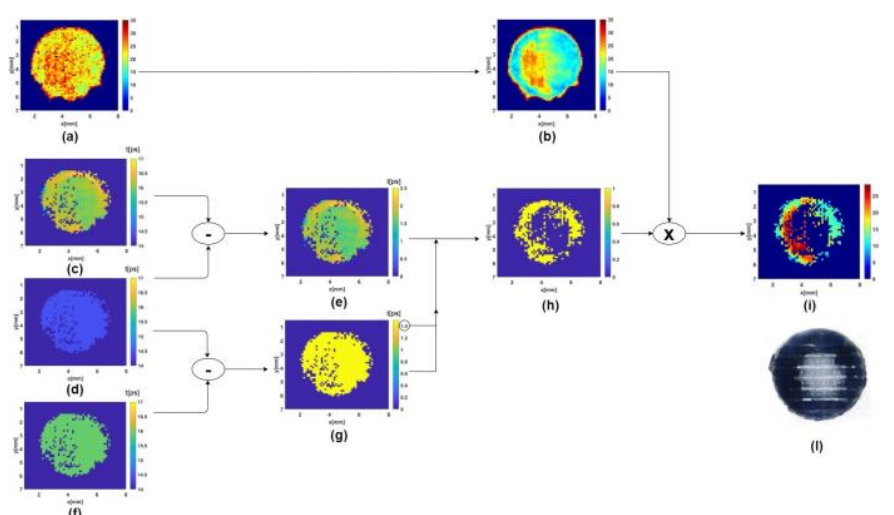

Fig. 13. Example of the developed $\mathrm{THz}$ data processing applied to the sample PCL15: a) raw $\mathrm{THz}$ data in false color. b) $\mathrm{THz}$ data after band pass and singular value decomposition filters. c) $2 \mathrm{D}$ map of the propagation time distribution (in picoseconds) in a magnetic scaffold (PCL-FeOx15). d) propagation time for the reference blank scaffold. e) time delay difference. f) sample loaded homogeneously with MNCs. g) threshold mask. h) 2D magnetization map.

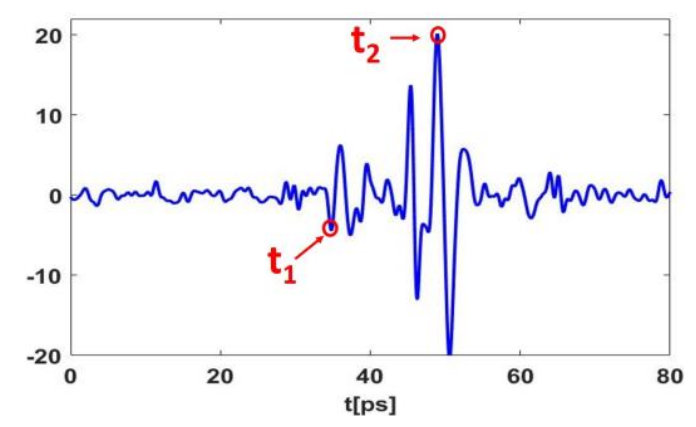

Fig. 14. Average A-scan. The time $t$ that the waveform employs to propagate inside the object is given by $t=t_{2}-t_{1}$ where $t_{1}$ corresponds to the beginning of the scaffold (air/object interface) and $t_{2}$ corresponds to the temporal position of the last peak due to object/support interface.

First, the propagation time of the $\mathrm{THz}$ signal within the sample under test is compared with the one that would be measured in the reference conditions (that is without MNCs). For the sample under test, the propagation time has been evaluated, for each pixel of the scanned area intercepting the sample, as the values ranging from $t_{0}=0$ corresponding to the time location of the first peak (due to the air/scaffold interface) and $t_{f}$ corresponding to the position of the last peak (due to the object/support interface, see Fig. 15).

This propagation time depends on the material wherein the signal propagation occurs and it is therefore affected by the presence and the amount of the MNCs. For reference, the propagation time has been computed by considering a homogeneous object having the same thickness of the scaffold under test and the refractive index estimated for the sample M0, i.e. the scaffold without MNCs $(n=1.73$ as shown in Tab. I). Finally, by subtracting (pixel by pixel) the reference propagation time from the propagation time appraised in the sample under test, a 2D differential map was obtained. This map is referred to as a propagation time delay map. For example, Fig. 13.c shows the 2D map of the propagation time distribution (in picoseconds) of the measured signal of PCL FeOx 15 sample, while Figure 13.d shows the propagation time for the reference and Fig. 13.e the time delay difference. 


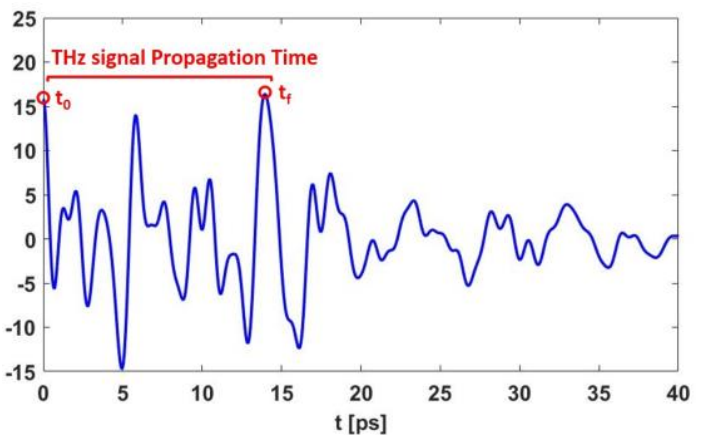

Fig. 15. Average THz signal collected for a single pixel: $t_{0}$ corresponds to the temporal position of the first peak due to the air/object interface, $t_{f}$ corresponds to the temporal position of the last peak due to object/support interface. $\mathrm{THz}$ signal propagation time in the sample analyzed at the single-pixel discretizing the scan area is evaluated as the difference between $t_{f}$ and $t_{0}$.

The third step of the MNPs Distribution Estimate deals with the identification of a threshold value that allows the detection of the areas of the samples characterized by the presence of MNPs. To set this threshold value, another reference image is generated by computing the $t$ that would be measured in a sample homogeneously loaded with MNC (Fig. 13.f). This value is derived using the TOF formula (2) where $d$ is the thickness of the sample under test and $v$ is the electromagnetic wave velocity in an object having the refractive index estimated for the scaffold under test (see Tab. I). Then, a threshold mask is obtained by taking the difference between the two references propagation times, i.e., the times of flight for the reference sample with and without MNPs (see Fig. 13.g). This mask is used to threshold the propagation time delay map (Fig. 13.e) and to obtain a binary magnetization map (Fig. 13.h). In this image, the pixels where the propagation time is larger than the threshold are set to one being the pixel where the presence of the MNCs most significantly affects the $\mathrm{THz}$ signal during its propagation through the sample. The binary magnetization map is the output of the Threshold Estimate step and allows also the estimate of a Magnetization Index $\left(I_{m}\right)$, synthetically encoding the amount of MNCs present in the sample under test (for a homogeneous reference). The $I_{m}$ value has been calculated as the ratio between the number of pixels containing MNPs (yellow pixels marked with "1" in Fig. 13.h) and the total number of pixels discretizing the scanning area and intercepting the sample. Table I shows the values of $I_{m}$ for all the scaffolds analyzed. Finally, in the MNCs Distribution Step, a 2D MNCs distribution map (Fig. 13.i) is obtained by multiplying pixel by pixel the binary magnetization map times (Fig. 13.h) and the map of the filtered $\mathrm{THz}$ signal amplitude (Fig. 13.b). Fig. 13.i shows the parts of the sample under test where MNPs are mostly concentrated and are used to evaluate the potential of the manufactured magnetic scaffolds for the hyperthermia treatment of bone tumors.

\section{ACKNOWLEDGMENTS}

The authors acknowledge CeSAR (Centro Servizi Ricerca d'Ateneo) core facility of the University of Cagliari and Dr. A. Ardu for assistance with the generation of images on JeolJEM 1400 Plus.

The authors thank the Materials Characterization and
Electron Microscopy Facilities of the Italian Institute of Technology for support with material characterization.

This work is relevant to the aims and objectives of WG2 from COST Action 17115 MyWave

This work has been supported by PRIN BEST-Food Broadband Electromagnetic Sensing Technologies for Food quality and security assessment (grant n. 20179FLH4A).

\section{REFERENCES}

[1] N. R. Datta, S. Gomez Ordo'nez, U. S. Gaipl, M. M. Paulides, Hans Crezee, J. Gellermann, D. Marder, E. Puric, S. Bodis, "Local hyperthermia combined with radiotherapy and-/or chemotherapy: Recent advances and promises for the future," Cancer Treatment Reviews, vol. 41, no. 9, pp. 742-753, 2015.

[2] M. D. Hurwitz, "Hyperthermia and immunotherapy: clinical opportunities," International Journal of Hyperthermia, vol. 36, no. 1, pp. 4-9, 2019

[3] N. R. Datta, H. P. Kok, H. Crezee, U. S. Gaipl, S. Bodis, "Integrating loco-regional hyperthermia into the current oncology practice: SWOT and TOWS analyses," Frontiers in Oncology, vol. 10, 2020.

[4] S.-Y. Lee, G. Fiorentini, A. M. Szasz, G. Szigeti, A. Szasz, C. A. Minnaar, "Quo Vadis Oncological Hyperthermia (2020)?," Frontiers in Oncology, vol. 10, 2020.

[5] E. Skafida, S. Kokkali, M. Nikolaou, A. Digklia, "Metastatic soft tissue sarcoma: Current treatment landscape and future perspectives," Expert Reviews in Anticancer Therapy, vol. 17, no. 6, pp. 537-543, 2017

[6] T. F. DeLaney, L. Park, I. S. Goldberg, E.B. Hug, N. J. Liebsch, K. E. Munzenrider, H. D. Suit, "Radiotherapy for local control of osteosarcoma," International Journal of Radiation Oncology, Biology and Physics, vol. 61, no. 2, pp. 492-498, 2005.

[7] A. Luetke, et al., "Osteosarcoma treatment-where do we stand? A state of the art review," Cancer Treatment Reviews, vol. 40, no. 4, pp. 523532, 2014

[8] Y. Yang, L. Han, Z. He, X. Li, S. Yang, Y. Zhang, D. li, Y. Yang, Z. Yang, "Advances in limb salvage treatment of osteosarcoma," J. Bone Oncol., 10, pp. 36-40, 2018.

[9] C. Meazza, S. Bastoni, P. Scanagatta, "What is the best clinical approach to recurrent/refractory osteosarcoma?," Expert Review of Anticancer Therapy, vol. 20, no. 5, pp. 415-428, 2020.

[10] V. Carina, V. Costa, M. Sartori, D. Bellavia, A. De Luca, L. Raimondi,M. Fini, G. Giavaresi,"Adjuvant biophysical therapies in osteosarcoma," Cancers, vol. 11, no. 3, 348, 2019.

[11] Q.-Y. Fan, B.-A. Ma, X.-C. Qiu, Y.-L. Li, J. Ye, and Y. Zhou, "Preliminary report on treatment of bone tumors with microwaveinduced hyperthermia," Bioelectromagnetics, vol. 17, no. 3, pp. 218222, 1996.

[12] Q.-Y. Fan, B.-A. Ma, Y. Zhou, M.-H. Zhang, and X.-B. Hao, "Bone tumors of the extremities or pelvis treated by microwave-induced hyperthermia," Clin. Orthopaedics Related Res., vol. 406, no. 1, pp. 165-175, 2003.

[13] Q.-Y. Fan, et al., "Microwave ablation of malignant extremity bone tumors," SpringerPlus, vol. 5, no. 1, pp. 1-6, 2016.

[14] K. Han, et al., "Is limb salvage with microwave-induced hyperthermia better than amputation for osteosarcoma of the distal tibia?," Clinical Orthopaedics and Related Research ${ }^{\circledR}$, vol. 475, no. 6, pp. 1668-1677, 2017.

[15] S. Behrens, "Preparation of functional magnetic nanocomposites and hybrid materials: recent progress and future directions," Nanoscale, vol. 3, no. 3, pp. 877-892, 2011.

[16] Y. Li, G. Huang, X. Zhang, B. Li, Y. Chen, T. Lu, T. J. Lu, and F. Xu, "Magnetic hydrogels and their potential biomedical applications," Adv. Funct. Mat., vol. 23, no. 6, pp. 660-672, 2013.

[17] A. Baeza, D. Arcos, M. Vallet-Reg '1, "Thermoseeds for interstitial magnetic hyperthermia: from bioceramics to nanoparticles," J. Phys. Cond. Mat., vol. 25, no. 48, p. 484003, 2013.

[18] H.-Y. Xu, N. Gu, "Magnetic responsive scaffolds and magnetic fields in bone repair and regeneration," Front. Mat. Sci., vol. 8, no. 1, pp. 20-31, 2014.

[19] A. A. Adedoyin, A. K. Ekenseair, "Biomedical applications of magnetoresponsive scaffolds," Nano Res., vol. 11, no. 10, pp. 50495064, 2018. 
[20] B. Smolkova, M. Uzhytchak, A. Lynnyk, S. Kubinova, A. Dejneka, O. Lunov, "A critical review on selected external physical cues and modulation of cell behavior: Magnetic nanoparticles, non-thermal plasma and lasers," J. Fun. Biomat., vol. 10, no. 1, p. 2, 2019.

[21] M. Miola, Y. Pakzad, S. Banijamali, S. Kargozar, C. Vitale-Brovarone, A. Yazdanpanah, O. Bretcanu, A. Ramedani, E. Verne, and M. Mozafari, "Glass-ceramics for cancer treatment: so close, or yet so far?" Acta Biomaterialia, vol. 83, pp. 55-70, 2019.

[22] C. Wu, W. Fan, Y. Zhu, M. Gelinsky, J. Chang, G. Cuniberti, V. Albrecht, T. Friis, and Y. Xiao, "Multifunctional magnetic mesoporous bioactive glass scaffolds with a hierarchical pore structure," Acta Biomaterialia, vol. 7, no. 10, pp. 3563-3572, 2011.

[23] J. Zhang, S. Zhao, M. Zhu, Y. Zhu, Y. Zhang, Z. Liu, and C. Zhang, "3d-printed magnetic fe $304 / \mathrm{mbg} / \mathrm{pcl}$ composite scaffolds with multifunctionality of bone regeneration, local anticancer drug delivery and hyperthermia," J. Mat. Chem. B, vol. 2, no. 43, pp. 7583-7595, 2014.

[24] A. Farzin, M. Fathi, and R. Emadi, "Multifunctional magnetic nanostructured hardystonite scaffold for hyperthermia, drug delivery and tissue engineering applications," Mat. Sci. Eng.: C, vo. 70, pp. 21-31, 2017.

[25] M. Fernandes, D. Correia, A. da Costa, S. Ribeiro, M. Casal, S. Lanceros-Mendez, and R. Machado, "Multifunctional magnetically re-' sponsive biocomposites based on genetically engineered silk-elastin like protein," Composites Part B: Engineering, vol. 153, pp. 413-419, 2018.

[26] M. Miola, A. Bellare, F. Laviano, R. Gerbaldo, and E. Verne, "Bioactive superparamagnetic nanoparticles for multifunctional composite bone cements," Cer. Intern., vol. 45, no. 12, pp. 14 533-14 545, 2019.

[27] S. Sprio, E. Campodoni, M. Sandri, L. Preti, T. Keppler, F. A. Muller, N. M. Pugno, A. Tampieri, "A graded multifunctional hybrid scaffold with superparamagnetic ability for periodontal regeneration," International Journal of Molecular Sciences, vol. 19, no. 11, p. 3604, 2018.

[28] C. Shuai, Y. Cheng, W. Yang, P. Feng, Y. Yang, C. He, F. Qi, S. Peng, "Magnetically actuated bone scaffold: Microstructure, cell response and osteogenesis", Composite Part B, vol. 192, 107986, 2020.

[29] Y. Sapir-Lekhovitser, M. Y. Rotenberg, J. Jopp, G. Friedman, B. Polyak, S. Cohen, "Magnetically actuated tissue engineered scaffold: insights into mechanism of physical stimulation," Nanoscale, vol. 8, no. 6, pp

[30] P. S. Castro, M. Bertotti, A. F. Naves, L. H. Catalani, D. R. Cornejo, G. D. Bloisi, and D. F. Petri, "Hybrid magnetic scaffolds: The role of scaffolds charge on the cell proliferation and $\mathrm{Ca} 2+$ ions permeation," Coll Surf B: Bioint., vol. 156, pp. 388-396, 2017.

[31] L. Chen, J. Peng, J. Zhao, Y. Long, Y. Xie, and J. Nie, "Magnetic materials in promoting bone regeneration," Front. Mat., vol. 6, p. 268, 2019.

[32] U. D'Amora, T. Russo, A. Gloria, V. Rivieccio, V. D’Anto, G. Negri, L. Ambrosio, R. De Santis, "3d additive-manufactured nanocomposite magnetic scaffolds: Effect of the application mode of a time-dependent magnetic field on hmscs behavior," Bioact. Mat., vol. 2, no. 3, pp. 138145, 2017.

[33] M. T. Lopez-Lopez, G. Scionti, A. C. Oliveira, J. D. Duran, A. Campos, M. Alaminos, I. A. Rodriguez, "Generation and characterization of novel magnetic field-responsive biomaterials," PLoS One, vol. 10, no. 7, p. e0133878, 2015.

[34] H.-M. Yun, S.-K. Kang, R. K. Singh, J.-H. Lee, H.-H. Lee, K.-R. Park, J.-K. Yi, D.-W. Lee, H.-W. Kim, E.-C. Kim, "Magnetic nanofiber scaffold-induced stimulation of odontogenesis and pro-angiogenesis of human dental pulp cells through wnt/mapk/nf-kb pathways," Dental Mat., vol. 32, no. 11, pp. 103943-103955, 2016.

[35] M. Filippi, B. Dasen, J. Guerrero, F. Garello, G. Isu, G. Born, M. Ehrbar, I. Martin, and A. Scherberich, "Magnetic nanocomposite hydrogels and static magnetic field stimulate the osteoblastic and vasculogenic profile of adipose-derived cells," Biomaterials, vol. 223, p. 119468, 2019.

[36] K. M. Sajesh, A. Ashokan, G. S. Gowd, T. B. Sivanarayanan, A. Unni, S. V. Nair, and M. Koyakutty, "Magnetic 3d scaffold: A theranostic tool for tissue regeneration and non-invasive imaging in vivo," Nanomed.: Nanotech. Biol. Med., vol. 18, pp. 179-188, 2019.

[37] A. Tampieri, T. D’Alessandro, M. Sandri, S. Sprio, E. Landi, L. Bertinetti, S. Panseri, G. Pepponi, J. Goettlicher, M. Banobre-Lopez, "Intrinsic magnetism and hyperthermia in bioactive fe-doped hydroxyapatite," Acta Biomaterialia, vol. 8, no. 2, pp. 843-851, 2012.

[38] R. K. Singh, M. Srivastava, N. Prasad, P. Shetty, and S. Kannan, "Hyperthermia effect and antibacterial efficacy of fe3+/co2+ cosubstitutions in $\beta$-Ca3(PO4)2 for bone cancer and defect therapy," Journal of Biomedical Materials Research Part B: Applied Biomaterials, vol. 106, no. 3, pp. 1317-1328, 2018

[39] S. Sahmani, A. Khandan, S. Saber-Samandari, M. Aghdam, "Vibrations of beam-type implants made of $3 \mathrm{~d}$ printed bredigite magnetite bionanocomposite scaffolds under axial compression: Application, communication and simulation," Ceramics International, vol. 44, no. 10 , pp. $11282-11291,2018$.

[40] K. Lai, W. Jiang, J. Z. Tang, Y. Wu, B. He, G. Wang, Z. Gu, "Superparamagnetic nano-composite scaffolds for promoting bone cell proliferation and defect reparation without a magnetic field," RSC Adv, vol. 2, no. 33, pp. 13 007-13 017, 2012.

[41] H. Zhang, J. Xia, X. Pang, M. Zhao, B. Wang, L. Yang, H. Wan, J. Wu, S. Fu, "Magnetic nanoparticle-loaded electrospun polymeric nanofibers for tissue engineering," Material Science Engineering: C, vol. 73, pp. 537-543, 2017.

[42] M. Banobre-Lopez, Y. Pineiro-Redondo, M. Sandri, A. Tampieri, R. De Santis, V. A. Dediu, J. Rivas, "Hyperthermia induced in magnetic scaffolds for bone tissue engineering," IEEE Transaction on Magnetics, vol. 50, no. 11, pp. 1-7, 2014

[43] A. Riminucci, C. Dionigi, C. Pernechele, G. De Pasquale, T. De Caro, G. M. Ingo, F. Mezzadri, N. Bock, M. Solzi, G. Padeletti, M. Sandri, A. Tampieri, V. Dediu, "Magnetic and morphological properties of ferrofluid-impregnated hydroxyapatite/collagen scaffolds," Sci. $A d v$. Mat., vol. 6, no. 12, pp. 2679-2687, 2014.

[44] S. El-Dek, M. A. Ali, S. M. El-Zanaty, and S. E. Ahmed, "Comparative investigations on ferrite nanocomposites for magnetic hyperthermia applications," Journal of Magnetism and Magnetic Materials, vol. 458, pp. $147-155,2018$.

[45] N. Bock, A. Riminucci, C. Dionigi, A. Russo, A. Tampieri, E. Landi, V. A. Goranov, M. Marcacci, and V. Dediu, "A novel route in bone tissue engineering: magnetic biomimetic scaffolds," Acta Biomaterialia, vol. 6, no. 3, pp. 786-796, 2010.

[46] X. B. Zeng, H. Hu, L. Q. Xie, F. Lan, W. Jiang, Y. Wu, and Z. W. Gu, "Magnetic responsive hydroxyapatite composite scaffolds construction for bone defect reparation," International Journal of Nanomedicine, vol. 7, p. 3365, 2012.

[47] S. K. Samal, M. Dash, T. Shelyakova, H. A. Declercq, M. Uhlarz, M. Banobre-Lopez, P. Dubruel, M. Cornelissen, T. Herrmannsdorfer, J. Rivas et al., "Biomimetic magnetic silk scaffolds," ACS Applied Materials \& Interfaces, vol. 7, no. 11, pp. 6282-6292, 2015

[48] M. B. Lodi, A. Fanti, G. Muntoni, G. Mazzarella, "A Multiphysic Model for the Hyperthermia Treatment of Residual Osteosarcoma Cells in Upper Limbs Using Magnetic Scaffolds, IEEE J Multisc. Multiph. Comp. Tech., vol. 4, pp.337-347, 2019

[49] P. R. Stauffer, T. C. Cetas, R. C. Jones, "Magnetic induction heating of ferromagnetic implants for inducing localized hyperthermia in deepseated tumors," IEEE Transactions on Biomedical Engineering, vol. 2, pp. 235-251, 1984

[50] A. Matsumine, et al., "A novel hyperthermia treatment for bone metastases using magnetic materials," International Journal of Clinical Oncology, vol. 16, no. 2, pp. 101-108, 2011.

[51] H. Dobsicek Trefna, et al., "Quality Assurance Guidelines for Interstitial Hyperthermia," International Journal of Hyperthermia, vol. 36, no. 1, pp. 276-293, 2019

[52] Y. Amemiya, A. Arakaki, S. S. Staniland, T. Tanaka, T. Matsunaga, "Controlled formation of magnetite crystal by partial oxidation of ferrous hydroxide in the presence of recombinant magnetotactic bacterial protein Mms6" Biomaterials, vol. 28, pp. 5381-5389, 2007.

[53] A. Fanti, M. B. Lodi, and G. Mazzarella, "Enhancement of cell migration rate toward a superparamagnetic scaffold using if magnetic fields," IEEE T Mag, vol. 52, no. 10, pp. 1-8, 2016.

[54] H. Hu, W. Jiang, F. Lan, X. Zeng, S. Ma, Y. Wu, Z. Gu, "Synergic effect of magnetic nanoparticles on the electrospun aligned superparamagnetic nanofibers as a potential tissue engineering scaffold," RSC Adv, vol. 3, no. 3, pp. 879-886, 2013.

[55] J.-J. Kim, R. K. Singh, S.-J. Seo, T.-H. Kim, J.-H. Kim, E.-J. Lee, H.W. Kim, "Magnetic scaffolds of polycaprolactone with functionalized magnetite nanoparticles: physicochemical, mechanical, and biological properties effective for bone regeneration," RSC Adv, vol. 4, no. 33, pp. 17325-17336, 2014.

[56] M. L. Mather, S. P. Morgan, L. J. White, H. Tai, W. Kockenberger, S. M. Howdle, K. M. Shakesheff, and J. A. Crowe, "Image-based characterization of foamed polymeric tissue scaffolds," Biomed Mat, vol. 3, no. 1, 015011, 2008. 
[57] I. Busboom, et al., "Terahertz Imaging of 3D Print Infill Structures," in 2021 15th European Conference on Antennas and Propagation (EuCAP). IEEE, 2021.

[58] Fukasawa, "Terahertz imaging: Widespread industrial application in non-destructive inspection and chemical analysis," IEEE T Terahertz Sci Tech, vol. 5, no. 6, pp. 1121-1127, 2015 .

[59] A. A. Mahmoud, A. H. Salama, "Norfloxacin-loaded collagen/chitosan scaffolds for skin reconstruction: Preparation, evaluation and in-vivo wound healing assessment," Eur J Pharm Sci, vol. 83, pp. 155-165, 2016

[60] J. Takayanagi, H. Jinno, S. Ichino, K. Suizu, M. Yamashita, T. Ouchi, S. Kasai, H. Ohtake, H. Uchida, N. Nishizawa et al., "High-resolution time-of-flight terahertz tomography using a femtosecond fiber laser," Optics Express, vol. 17, no. 9, pp. 7533-7539, 2009.

[61] I. Catapano and F. Soldovieri, "THz imaging and spectroscopy: First experiments and preliminary results," in 2015 8th International Workshop on Advanced Ground Penetrating Radar (IWAGPR). IEEE, 2015, pp. 1-4.

[62] Catapano and Soldovieri, "A data processing chain for terahertz imaging and its use in artwork diagnostics," Journal of Infrared, Millimeter, and Terahertz Waves, vol. 38, no. 4, pp. 518-530, 2017.

[63] Zomega, “Thz fico system," 2015. [Online]. Available: http://www.optron.com/images/zomegacatalogue1401lr.pdf

[64] A. Fanti, M. B. Lodi, G. Vacca, and G. Mazzarella, "Numerical investigation of bone tumor hyperthermia treatment using magnetic scaffolds," IEEE J Electromag RF Microw Med Biol, vol. 2, no. 4, pp. 294-301, 2018.

[65] M. Colombo, et al., "Biological applications of magnetic nanoparticles," Chemical Society Reviews, vol. 41, no. 11, pp. 4306-4334, 2012.

[66] M. Miola, et al., "Bioactive superparamagnetic nanoparticles for multifunctional composite bone cements," Ceramics International, vol. 45, no. 12, pp. 14533-14545, 2019.

[67] J. Li, et al. "Numerical simulation of magnetic fluid hyperthermia based on multiphysics coupling and recommendation on preferable treatment conditions," Current Applied Physics, vol. 19, no. 9, pp. 1031-1039, 2019

[68] A. G. Kolhatkar, et al., "Tuning the magnetic properties of nanoparticles.," International journal of Molecular Sciences, vol. 14, no. 8, pp. 15977-16009.

[69] R. A. M. Canters, et al. "A literature survey on indicators for characterisation and optimisation of SAR distributions in deep hyperthermia, a plea for standardization," International Journal of Hyperthermia, vol. 25, no. 7, pp. 593-608, 2009.

[70] M. M. Paulides, et al. "Simulation techniques in hyperthermia treatment planning," International Journal of Hyperthermia, vol. 29, no. 4, pp. 346-357, 2013

[71] G. G. Bellizzi, et al,. "The potential of constrained SAR focusing for hyperthermia treatment planning: Analysis for the head \& neck region," Physics in Medicine \& Biology, vol. 64, no. 1, 015013, 2018.

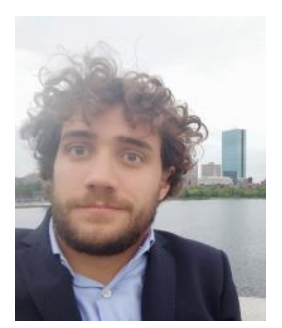

Matteo B. Lodi (Graduate Student Member) received the bachelor's degree in biomedical engineering from the University of Cagliari, Cagliari, in 2016, and the master's degree in biomedical engineering from Politecnico di Torino, Turin, Italy, in 2018. He is currently working toward the Ph.D. degree in electronic engineering and computer science at the University of Cagliari. His research activity deals with the modeling of bioelectromagnetic phenomena, especially hyperthermia treatment; the study, manufacturing, and synthesis of magnetic biomaterials for tissue engineering applications; and the use of microwave for biotechnology and environmental applications. He was awarded as Young Scientists at General Assembly and Scientific Symposium of URSI 2020 and 2021. He has been appointed as Representative for the Young Professionals of IEEE Region 8 Nanotechnology Council. He is a member of the Editorial Board of the IEEE Future Directions Technology Policy and Ethics newsletter.

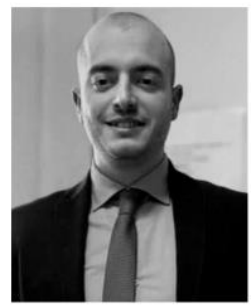

Nicola Curreli after the M.Sc. degree from the University of Genoa (Genoa, Italy), in 2016, received the Ph.D. degree in Electronic Engineering, from the University of Cagliari (Cagliari, Italy) and the Italian Institute of Technology - IIT (Genoa, Italy), in 2020. In 2019 he was visiting researcher with the Physics and Mechanical Engineering Departments at Columbia University in the City of New York (New York, USA). After the Ph.D., he held a fellow position at the Graphene Labs - IIT within the WP12 (Energy storage) of the Graphene Core 2 project - Graphene flagship. He is currently a post-doc at the Functional Nanosystems group - IIT. His research activity is focused on the study of low-dimensional materials and their application in the fields of electronics and photonics.

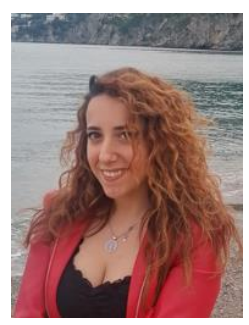

Sonia Zappia received the master's degree in biomedical engineering in 2019, and she is currently a PhD Student degree in Information Technologies and Electrical Engineering, both at the University of Naples Federico II, Italy. In the framework of her $\mathrm{PhD}$ project, she started her activity at the Institute for Electromagnetic Sensing of the Environment, National Research Council of Italy (IREA-CNR). Her research activity involves imaging with terahertz (THz) timedomain signals and $\mathrm{THz}$ spectroscopy. In particular, she focuses her work on the adoption of $\mathrm{THz}$ technology in food quality control and its exploitation as a tool for nondestructive inspection of magnetic biomaterials for tissue engineering applications.

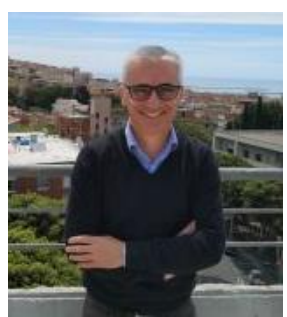

Luca Pilia is currently Associate Professor in the field of "Chemical Technology Fundamentals" (SSD CHIM/07) at the University of Cagliari, Italy. Since 2013 he has carried out his didactic and scientific activity at the Mechanical, Chemical and Material Engineering Department; he teaches Chemistry at the Faculty of Engineering. $\mathrm{He}$ graduated (with honors) in Chemistry at the University of Cagliari and took his $\mathrm{PhD}$ in Chemistry in 2004 at the same university. The research activity during the $\mathrm{PhD}$ was developed in collaboration with the group of Dr. Patrick Cassoux at the "Laboratoire de Chimie de Coordination du CNRS" (Toulouse, France) working on multifunctional materials based on transition metal complexes and organic donors. From 2003 to 2010 he was appointed as research assistant at the Department of Inorganic Chemistry, University of Cagliari, and from 2010 to 2012 he was postdoctoral researcher as Marie Curie IEF fellow and EPSRC research assistant at the School of Chemistry, University of Edinburgh (U. K.).

His main expertises are related to the synthesis and characterization of ligands with $\mathrm{O}, \mathrm{N}$ and $\mathrm{S}$ as donor atoms, and their complexes with transition metals. His research interest are focused on the metal complexes mainly of $\mathrm{Ni}(\mathrm{II}), \mathrm{Pd}(\mathrm{II}), \mathrm{Pt}(\mathrm{II})$ and $\mathrm{Fe}(\mathrm{II} / \mathrm{III})$ as precursors of materials with conducting and magnetic properties. The main L.P. interest is related to heteroleptic charge transfer metal complexes showing second order nonlinear optical (NLO) properties.

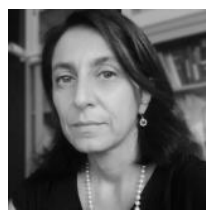

Maria Francesca Casula received the degree in Chemistry (summa cum laude) in 1998 and the PhD in Chemistry in 2002 from the University of Cagliari. Currently, she is Associate Professor of Chemical Fundamentals of Technologies at the Department of Mechanical, Chemical and Materials Engineering of the University of Cagliari, Italy. Her research is in the field of the preparation and characterization of functional materials, including nanocrystals, nanoporous solids and nanocomposites, for prospective application in biomedicine, catalysis, and environment. 


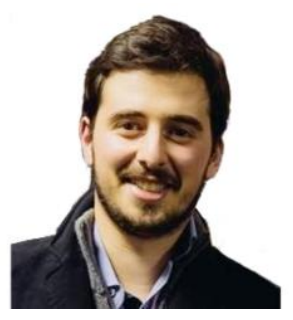

Sergio Fiorito Received his M.Sc. in Chemistry in 2016 and a Ph.D. in Nanochemistry in 2020 from the University of Genoa. During his Ph.D. he carried out his research activity at the Italian Institute of Technology, working in the Nanomaterial for Biomedical Applications research line. His work focused on the development of multi domain nano-heterostructures able to combine magnetic hyperthermia with radiation therapy and photothermal ablation in order to improve cancer treatments. He is currently a postdoctoral researcher in the Photonic Nanomaterials research line of the Italian Institute of Technology were he deals with the synthesis and post-synthesis functionalization of semiconductor nanocrystals in order to obtain new single-photon sources.

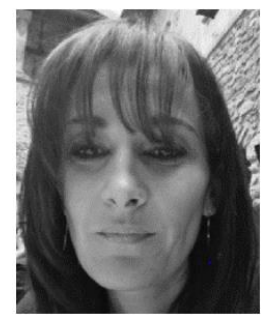

Ilaria Catapano received the Ph.D. degree in electric and information engineering from the University of Cassino, Cassino, Italy, in 2006. In 2003, in the framework of her Ph.D. project, she started her research activity with the Institute for Electromagnetic Sensing of the Environment, National Research Council of Italy (IREA-CNR), Naples, Italy, where she is a full-time Researcher. She was a Post-Doctoral Researcher with the Institute Fresnel of Marseille, Marseille, France, from 2006 to 2007. She was an Adjunct Professor of electromagnetic diagnostic with the Mediterranea University of Reggio Calabria, Reggio Calabria, Italy, in 2010 and an Invited Lecturer with Sao Paulo University, São Paulo, Brazil, in 2013. She joined IIT Kharagpur, Kharagpur, India, in 2016. She has coauthored over 150 articles, mainly in scientific journals or proceedings of international conferences and has been a reviewer for several international journals and conference. Her research activities deal with noninvasive electromagnetic diagnostics and are mainly focused on models and strategies for electromagnetic forward and inverse scattering problems, development and performance assessment of microwave imaging approaches for shape reconstruction, processing of experimental data gathered by radar systems for subsurface surveys, and $\mathrm{THz}$ spectroscopy and imaging.

Dr. Catapano received the G. Barzilai Award from the Italian Electromagnetic Society in 2004; she was one of the young scientist awardees at the XXIX URSI General Assembly in 2008

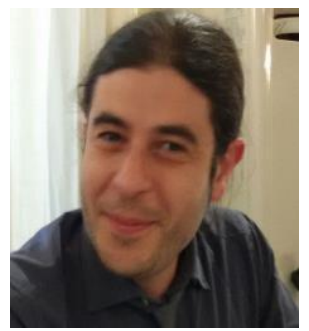

Francesco Desogus received the master's (with honors) degree in chemical engineering and the Ph.D. degree in industrial engineering from the University of Cagliari, Cagliari, Italy, in 2005 and 2010, respectively. Since 2014, he has been an Assistant Professor with the Department of Mechanical, Chemical and Materials Engineering, University of Cagliari. He taught courses of organic chemistry, industrial and energy processes, chemical plants, and transport phenomena. His current research interests include chemical and biological kinetics, chemical reactors, transport phenomena, biomass processing, food engineering and biomedical technologies.

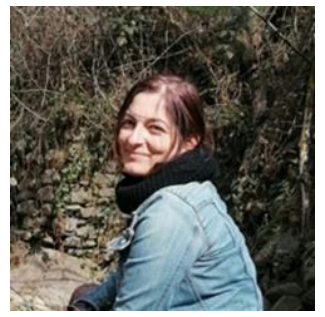

Teresa Pellegrino received the Laurea degree in Chemistry in 2000 and the $\mathrm{PhD}$ in Chemistry in 2005 from the University of Bari. She started working in nanoscience, after the Master when she was visiting student for 18 months in the group of Prof. $P$. Alivisatos at University of Berkeley (California) and later, during her $\mathrm{PhD}$, when she moved for additional 18 months in the group of Prof. W. J. Parak at the Center for Nanoscience in Munich (Germany). After being Post Doc at the National Nanotechnology Laboratory in Lecce (Italy), then permanent staff scientist at the Nanotech Center of CNR-Lecce, since 2014 she is tenured team leader of the Nanomaterials for Biomedical Applications group at the Italian Institute of Technology, Genoa (Italy). Her research group at IIT covers research activity from the development of organicinorganic nanostructured materials to their cellular in vitro and in vivo preclinical studies for applications ranging from drug delivery, to magnetic hyperthermia, photo-thermal ablation and radiotherapy.

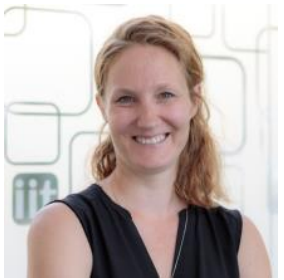

Ilka Kriegel started studying nanomaterials during her PhD at the Ludwig-MaximiliansUniversity in Munich, Germany accompanied by a research stay at the University of Chicago, IL, USA. During her first postdoc at the Politecnico di Milano, Italy, she got insight into the ultrafast spectral response of hybrid nanomaterials. A Marie-Curie (global) fellowship brought her to the Molecular Foundry, Berkeley, CA, USA and the Italian Institute of Technology (IIT), Genova, Italy, extending her knowledge to two-dimensional materials. Ilka is now head of the Functional Nanosystems group at IIT holding an ERC Starting Grant (Light-DYNAMO). She is further the coordinator of a collaborative FET Proactive grant (LIGHT-CAP). Her major research interests lie in the exploitation of functional nanosystems for energy and environment. Ilka is mother of three children.

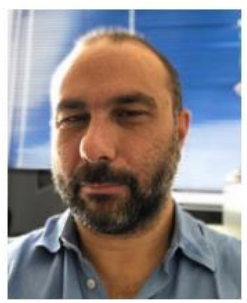

Lorenzo Crocco is a Senior Researcher with the Institute for the Electromagnetic Sensing of the Environment, National Research Council of Italy (IREA-CNR). His scientific activities mainly concern electromagnetic scattering, with a focus on diagnostic and therapeutic uses of EM fields, through-the-wall radar and GPR. On these topics, he has published more than 100 papers, given keynote talks and led or participated to research projects. $\mathrm{He}$ is associate editor for the IEEE Journal of Electromagnetics, RF and Microwaves in Medicine and Biology (IEEE J-ERM) and has edited a book on Electromagnetic Technologies for Brain Diseases Diagnostics, Monitoring and Therapy. From 2013 he is Italian representative in the Management Committees of COST actions devoted to medical applications of EM fields (MiMed on microwave imaging and MyWAVE on therapeutic applications of electromagnetic waves). Since 2017, he is Member of the Board of Directors of the Italian Electromagnetic Society (SIEm). Since 2019, he is a member of the Italian URSI Commission. In 2019, he has been elected in the Scientific Board of the Engineering Department (DIITET) of CNR. Dr. Crocco has been the recipient of the SIEm "Barzilai" Award for Young Scientists (2004) and YSA at the URSI General Assembly held in New Delhi (India) in 2005.

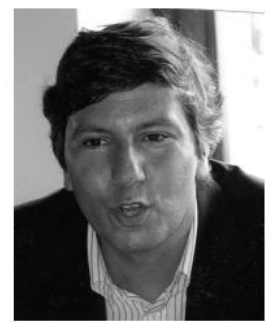

Giuseppe Mazzarella (Senior Member, IEEE) graduated with (summa with laude) in electronic engineering from the Universit Federico II of Naples, in 1984, and the Ph.D. degree in electronic engineering and computer science, in 1989. In 1990, he became an Assistant Professor at the Dipartimentodi Ingegneria Elettronica, Università Federico II of Naples. Since 1992, he has been with the Dipartimento dilngegneria Elettrica ed Elettronica of the Università di Cagliari, first as an Associate Professor and then, since 2000, as a Full Professor, teaching courses in electromagnetics, microwave, antennas and remote sensing. His research activity has focused mainly on: efficient design of large arrays of slots, power synthesis of array factor, with emphasis on inclusion of constraints, microwave holography techniques for the diagnosis of large reflector antennas, use of evolutionary programming for the solution of inverse problems, in particular problems of synthesis of antennas and periodic structures. $\mathrm{He}$ is author (or coauthor) of over 70 papers in international journals, and is a reviewer for many EM journals. 


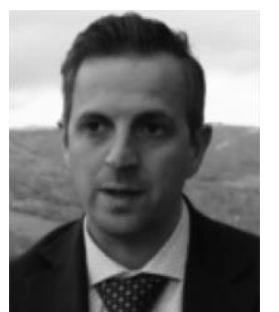

Alessandro Fanti (Member, IEEE) received the Laurea degree in electronic engineering and the Ph.D. degree in electronic engineering and computer science from the University of Cagliari, Cagliari, Italy, in 2006 and 2012, respectively. He worked as Postdoctoral Fellow in the Electromagnetic Group, University of Cagliari, from 2013 to 2016, where he is currently an Assistant Professor. His research activity involves the use of numerical techniques for modes computation of guiding structures, optimization techniques, analysis and design of waveguide slot arrays, analysis and design of patch antennas, radio propagation in urban environment, modeling of bio-electromagnetic phenomena, microwave exposure systems for biotechnology and bio-agriculture. $\mathrm{He}$ is an Associate Editor of the IEEE Journal of Electromagnetics, RF and Microwaves in Medicine and Biology (J-ERM). 


\section{Supplementary Material for \\ “Influence of Magnetic Scaffold Loading Patterns on their Hyperthermic Potential against Bone Tumors"}

\section{SM1. MNCs Characterization}

The two sets of magnetic nanocrystals (MNCs) were characterized by XRD and TEM. The results are presented in Fig. S1.
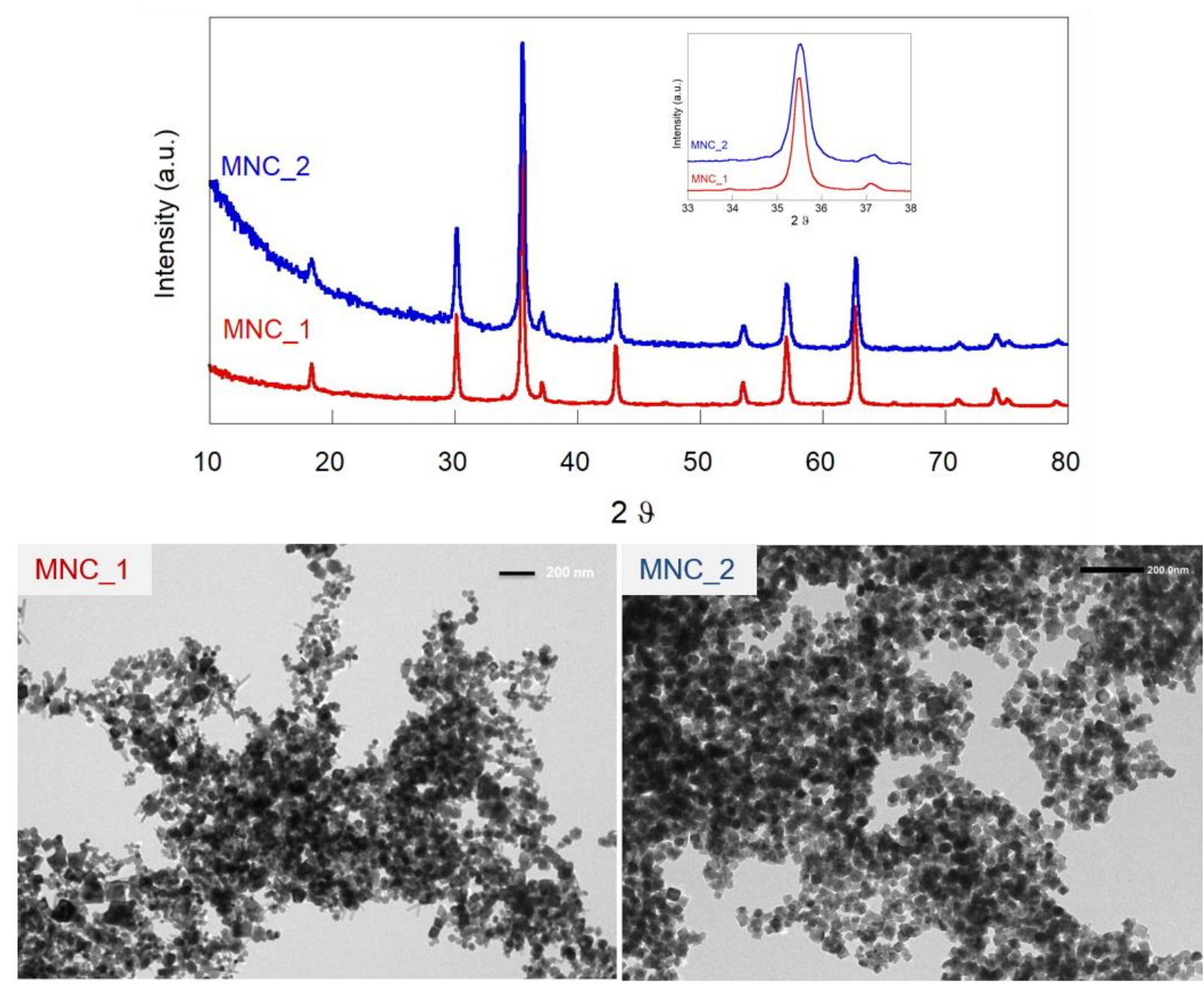

Fig. S1. XRD patterns (top) and corresponding representative TEM mages (bottom) of the iron oxide MNCs samples (MNC1, MNC2) used to produce PCL-based magnetic scaffolds.

\section{SM2. Dielectric and Thermal Properties of Tissues and Scaffolds}

\section{A. Initial Temperature}

The electromagnetic properties of scaffold and the tissues for the geometry shown in Fig. 5 in the manuscript, are reported in Tab. S1.

Table S1: Electromagnetic properties of MagS and tissues at $37^{\circ} \mathrm{C}$. 


\begin{tabular}{lcc}
\hline \hline & $\boldsymbol{\varepsilon}(\mathbf{3 0 0} \mathbf{~ k H z})$ & $\boldsymbol{\sigma}(\mathbf{3 0 0} \mathbf{~ k H z})$ \\
$\mathbf{S m}^{-\mathbf{1}}$
\end{tabular}

Table S2: Thermal properties of MagS and tissues at $37^{\circ} \mathrm{C}$.

\begin{tabular}{lcccc}
\hline \hline & $\mathbf{k}\left(\mathbf{W m}^{-1} \mathbf{K}^{-\mathbf{1}}\right)$ & $\mathbf{C}_{\mathbf{p}}\left(\mathbf{J ~ k g}_{\mathbf{1}} \mathbf{1}^{-\mathbf{K}}\right.$ & $\mathbf{Q}_{\mathbf{M}}\left(\mathbf{W} \mathbf{m}^{-\mathbf{3}}\right)$ & $\mathbf{\omega}_{\mathbf{B}}\left(\mathbf{s}^{\mathbf{- 1}}\right)$ \\
\hline $\mathbf{P C L}$ & 1.75 & 1300 & $/$ & $/$ \\
$\begin{array}{l}\text { Fracture } \\
\text { (inflamed) }\end{array}$ & 0.558 & 2450 & 5262.5 & $6.95 \mathrm{e}-3$ \\
$\begin{array}{l}\text { Fracture } \\
\text { (ischemic) }\end{array}$ & 0.1 & 2450 & 342.1 & $0.262 \mathrm{e}-3$ \\
Bone & 0.32 & 1313 & 286.2 & $0.175 \mathrm{e}-3$ \\
\hline \hline
\end{tabular}

\section{B. Temperature-dependence}

The magnetic, dielectric and thermal properties are assumed to be variable with temperature. The dielectric properties are assumed to linearly increase with temperature, with a variation of $3 \% \cdot{ }^{\circ} \mathrm{C}^{-1}$.

The thermal properties are assumed to linearly increase with a $1{ }^{\circ} \mathrm{C}$ increase of $0.5 \%$ for the thermal conductivities, whilst a $1{ }^{\circ} \mathrm{C}$ increase of $0.33 \%$ is assumed for the specific heat. The heat capacity of blood has been assumed linear with a $1 \% \cdot{ }^{\circ} \mathrm{C}^{-1}$.

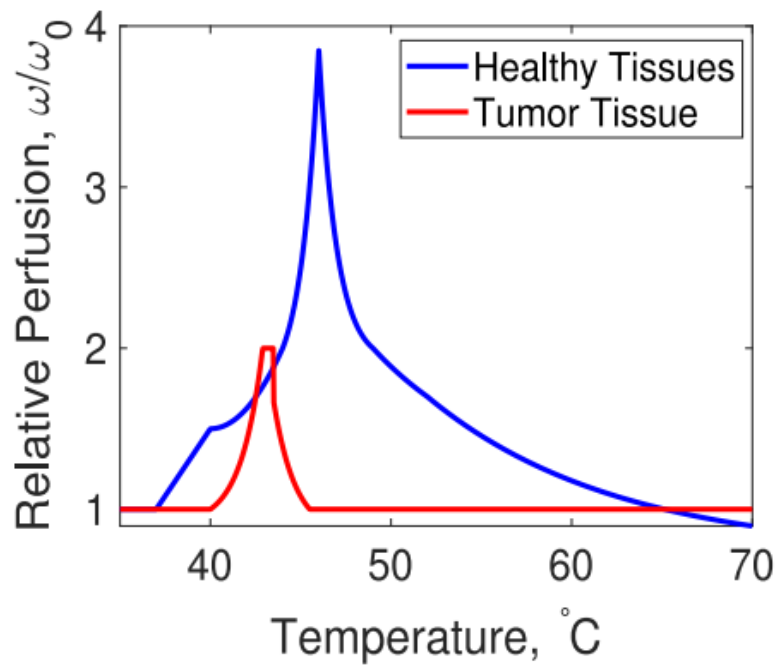


Fig. S3. Healthy and tumor tissues blood perfusion as a function of temperature, normalized to the value at $37^{\circ} \mathrm{C}$.

For the tissue perfusion, which is the outmost relevant physical characteristics which influences the outcome of the hyperthermia treatment, we used the piecewise function from [48] for healthy and tumor tissues and shown in Fig. S3.

\section{SM3. Distribution of MNCs}

The spatial distribution of magnetic nanocrystals (MNCs) in the biomaterial matrix, $\phi(x, y)$, is derived by $\mathrm{THz}$ tomography according to the processing steps presented in Sect. II.D and better described in the Appendix of the manuscript. $\mathrm{n}$ order to perform the simulations, the MNCs distribution is approximated to a finite series of radial basis functions (RBF), so that:

$$
\phi(x, y)=\sum_{i=1}^{n=8} a_{i} e^{-b_{i}\left[\sqrt{\left(x-x_{0, i}\right)^{2}+\left(y-y_{0, i}\right)^{2}}\right]^{2}}
$$

where $a_{i}$ is the maximum amplitude of the basis function, $b_{i}$ is the variance-related parameter and $x_{0 i}$ and $y_{0 i}$ are the centers of the $i$-th basis function. The values of the coefficients for the eight RBF are found by minimizing, in a least square sense, the difference between Eq. (SM1) using the "fit" function from Matlab 2021 (The MathWorks Inc., MA USA). The range for the coefficients are reported in Tab. S3.b

Table S3: Space of solution for the RBF coefficients.

\begin{tabular}{cccc}
\hline & Min. & Max. \\
\hline$a_{i}$ & 0 & 1 \\
$b_{i}$ & 0.001 & 20 \\
$x_{0 i}$ & -7 & 7 \\
$y_{0 i}$ & -7 & 7 \\
\hline
\end{tabular}

The examples and results for the ten drop-casted magnetic scaffolds are shown in Fig. S3 below, Tab. S4 and S5.

Table S4: Coefficients of the radial basis functions for the MNCs distribution of the ten magnetic scaffolds.

\begin{tabular}{ccccccccccc}
\hline & M1 & M2 & M3 & M4 & M5 & M6 & PCLFeOx14 & PCLFeOx15 & PCLFeOx16 & STRIP \\
\hline$a_{1}$ & 0.6491 & 0.5186 & $6.9235 \mathrm{e}-$ & 0.0787 & 0.5221 & 0.1133 & $2.3800 \mathrm{e}-14$ & 0.4211 & 0.4356 & 0.2891 \\
& & & 06 & & & & & & \\
$b_{1}$ & 10.4136 & 14.9995 & 14.9976 & 13.9251 & 13.7782 & 11.6231 & 7.5001 & 15.0000 & 2.5000 & 8.5510 \\
$x_{01}$ & 0.3267 & 0.2974 & 1.7797 & -0.0921 & 0.3565 & 0.8188 & $-4.0975 \mathrm{e}-43$ & 0.7726 & -0.1388 & 0.2581 \\
$y_{01}$ & 0.1114 & 0.0547 & 0.0063 & -0.2459 & -0.0518 & -0.0383 & $-5.3799 \mathrm{e}-46$ & 0.0266 & -0.6161 & -0.2336 \\
$a_{2}$ & 0.7142 & 0.5339 & 0.6000 & 0.1004 & 0.3383 & 0.2876 & 0.3787 & 0.5732 & $2.3590 \mathrm{e}-05$ & 0.4598 \\
$b_{2}$ & 15.0000 & 14.9987 & 14.4814 & 10.7910 & 14.7529 & 15.0000 & 7.2743 & 15.0000 & 2.4444 & 9.6028 \\
$x_{02}$ & -0.4818 & -0.2008 & -0.2498 & 0.0284 & 0.1712 & -0.6265 & -0.3949 & -0.4968 & 0.4838 \\
$y_{02}$ & -0.2643 & -0.2591 & -0.3881 & -0.0013 & -0.3163 & -0.3960 & -0.4575 & -0.5038 & 2.1418 \\
$a_{3}$ & 0.8031 & 0.4039 & 0.5222 & 0.0840 & 0.5373 & 0.4397 & 0.6371 & 0.5726 & $2.0406 \mathrm{e}-05$ & 0.105 \\
$b_{3}$ & 9.5546 & 14.9999 & 13.4286 & 10.7363 & 14.5658 & 7.5046 & 15.0000 & 15.0000 & 2.4447 & 7.4091
\end{tabular}




\begin{tabular}{|c|c|c|c|c|c|c|c|c|c|c|}
\hline$x_{03}$ & -0.0069 & -0.4438 & 0.0886 & 0.0270 & -0.3002 & -0.4335 & -0.7681 & -0.6271 & 0.4096 & 0.3233 \\
\hline$y_{03}$ & -0.1857 & 0.1064 & -0.4619 & 0.0042 & -0.3442 & -0.0230 & 0.0017 & 0.0040 & 2.1485 & -0.1319 \\
\hline$a_{4}$ & 0.3816 & 0.1267 & 0.7083 & 0.4143 & 0.1206 & 0.4328 & 0.3988 & 0.3842 & $6.2745 \mathrm{e}-05$ & 0.1809 \\
\hline$b_{4}$ & 15.0000 & 15.0000 & 14.8707 & 14.2194 & 13.7687 & 12.9715 & 15.0000 & 15.0000 & 2.4413 & 8.4831 \\
\hline$x_{04}$ & -0.7695 & -0.5635 & -0.3692 & -0.1092 & -0.5494 & -0.3251 & 0.2402 & -0.0310 & 1.0274 & 0.0961 \\
\hline$y_{04}$ & 0.0100 & -0.4554 & -0.0675 & -0.2377 & 0.1865 & -0.6275 & -0.6634 & -0.7643 & 2.0566 & -0.9855 \\
\hline$a_{5}$ & 0.0759 & 0.3799 & 0.7111 & 0.0158 & 0.2213 & 0.3500 & 0.7358 & 0.4167 & $1.5339 \mathrm{e}-04$ & 0.2869 \\
\hline$b_{5}$ & 14.9236 & 14.9991 & 15.0000 & 13.3170 & 13.4912 & 12.6463 & 13.6126 & 13.1265 & 2.4365 & 6.2276 \\
\hline$x_{05}$ & -0.9558 & 0.4548 & 0.2374 & 0.5056 & 0.3063 & 0.2697 & 0.4929 & 0.5163 & -1.3541 & -0.2491 \\
\hline$y_{05}$ & 0.3635 & -0.5129 & -0.1567 & -0.6296 & -0.0346 & -0.5738 & -0.2857 & -0.4656 & 2.1130 & -0.6567 \\
\hline$a_{6}$ & $\begin{array}{c}1.8852 \mathrm{e}- \\
04\end{array}$ & 0.0887 & 0.5222 & 0.4449 & 0.5626 & 0.2713 & 0.6306 & 0.4769 & $1.0011 \mathrm{e}-04$ & $\begin{array}{c}3.0191 \mathrm{e}- \\
04\end{array}$ \\
\hline$b_{6}$ & 14.9907 & 14.9998 & 14.7440 & 14.9270 & 13.9932 & 15.0000 & 13.6667 & 15.0000 & 2.1906 & 8.6070 \\
\hline$x_{06}$ & 1.9705 & -0.4037 & -0.1462 & -0.1833 & -0.0716 & -0.2631 & 0.4832 & 0.0081 & 1.7443 & -0.0235 \\
\hline$y_{06}$ & 0.6764 & 0.6613 & 0.5112 & 0.0618 & 0.0501 & 0.4666 & 0.1688 & 0.8018 & 0.4228 & $\begin{array}{c}3.6154 \mathrm{e}- \\
14\end{array}$ \\
\hline$a_{7}$ & $\begin{array}{c}2.9260 \mathrm{e}- \\
04\end{array}$ & 0.5882 & 0.5592 & 0.3875 & 0.4181 & 0.4883 & 0.5318 & 0.7066 & 0.0835 & 0.2470 \\
\hline$b_{7}$ & 14.9830 & 14.9971 & 15.0000 & 14.7654 & 12.4712 & 4.7160 & 8.5543 & 15.0000 & 2.5000 & 7.8635 \\
\hline$x_{07}$ & 1.9705 & -0.0764 & 0.2259 & 0.1496 & -0.2987 & 0.2370 & -0.1807 & -0.3173 & -0.1261 & -0.1374 \\
\hline$y_{07}$ & $\begin{array}{c}2.7078 \mathrm{e}- \\
06\end{array}$ & 0.2942 & 0.2562 & 0.0279 & 0.2975 & 0.2610 & 0.5444 & 0.4875 & 0.3239 & $\begin{array}{c}8.4750 \mathrm{e}- \\
07\end{array}$ \\
\hline$a_{8}$ & 0.4380 & $\begin{array}{c}6.3209 \mathrm{e}- \\
04\end{array}$ & $\begin{array}{c}1.0000 \mathrm{e}- \\
04\end{array}$ & 0.0098 & 0.0078 & $\begin{array}{c}1.6478 \mathrm{e}- \\
04\end{array}$ & $1.6195 \mathrm{e}-04$ & $1.0004 \mathrm{e}-04$ & 0.3967 & 0.0782 \\
\hline$b_{8}$ & 14.9995 & 14.8785 & 15.0000 & 12.7537 & 13.2334 & 14.9827 & 9.5435 & 15.0000 & 1.7464 & 3.4788 \\
\hline$x_{08}$ & 0.4000 & -0.0191 & 0.0880 & 0.0064 & 0.0372 & 0.0173 & $2.3800 \mathrm{e}-14$ & 0.4211 & -0.1042 & 0.2891 \\
\hline$y_{08}$ & 0.4377 & 1.7074 & 1.8186 & 0.0787 & 0.5221 & 0.1133 & 7.5001 & 15.0000 & 0.4356 & 8.5510 \\
\hline
\end{tabular}



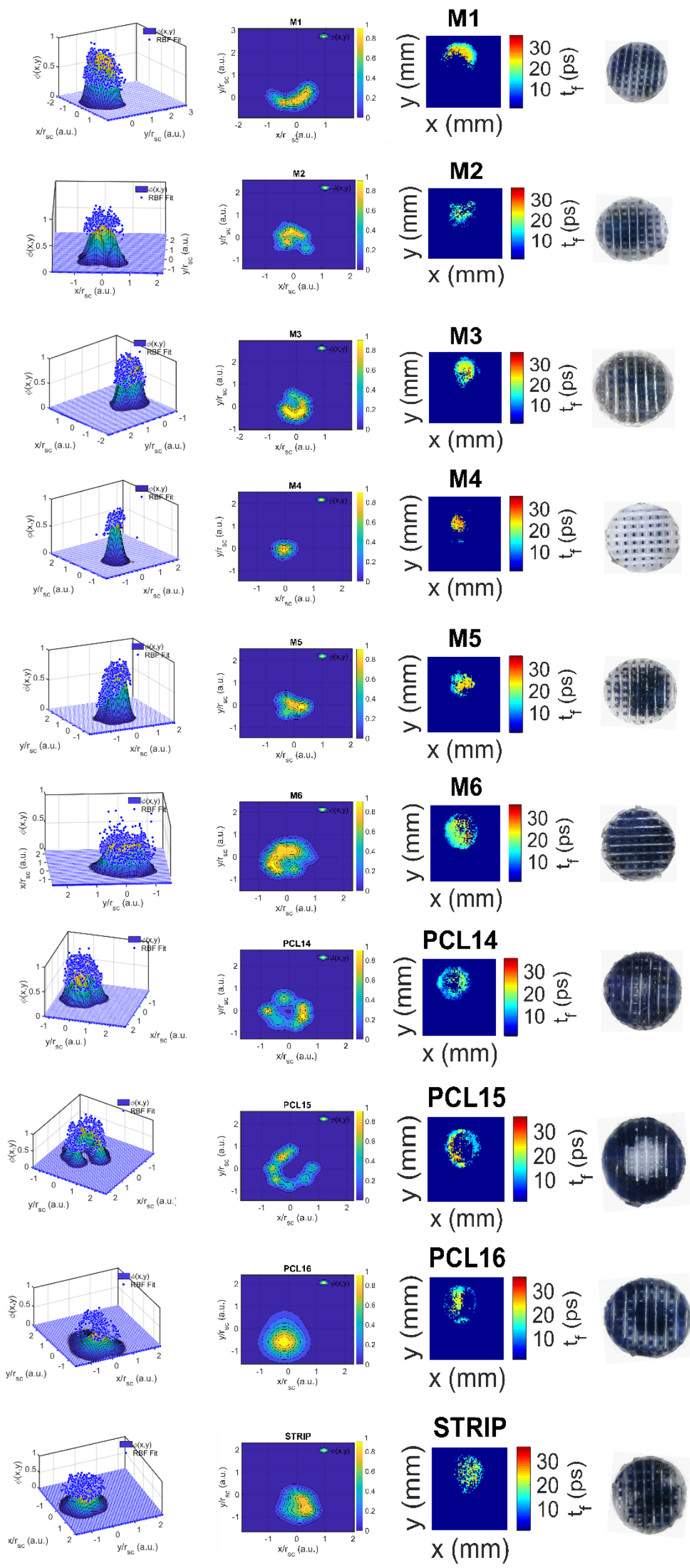
Fig. S3. Fittings results and comparison with THz scans and optical images.

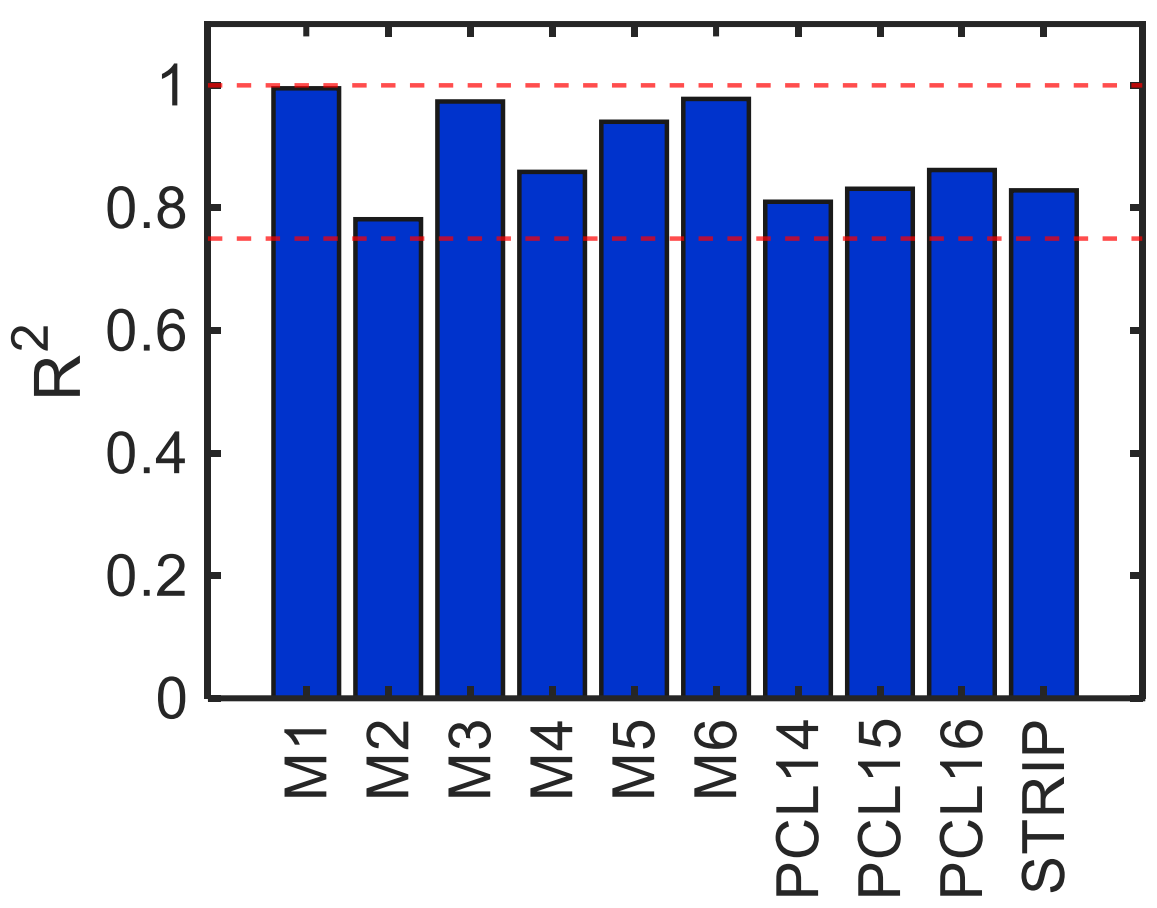

Fig. S4. Residuals $\left(R^{2}\right)$ for the fittings.

SM4. SEM Analysis
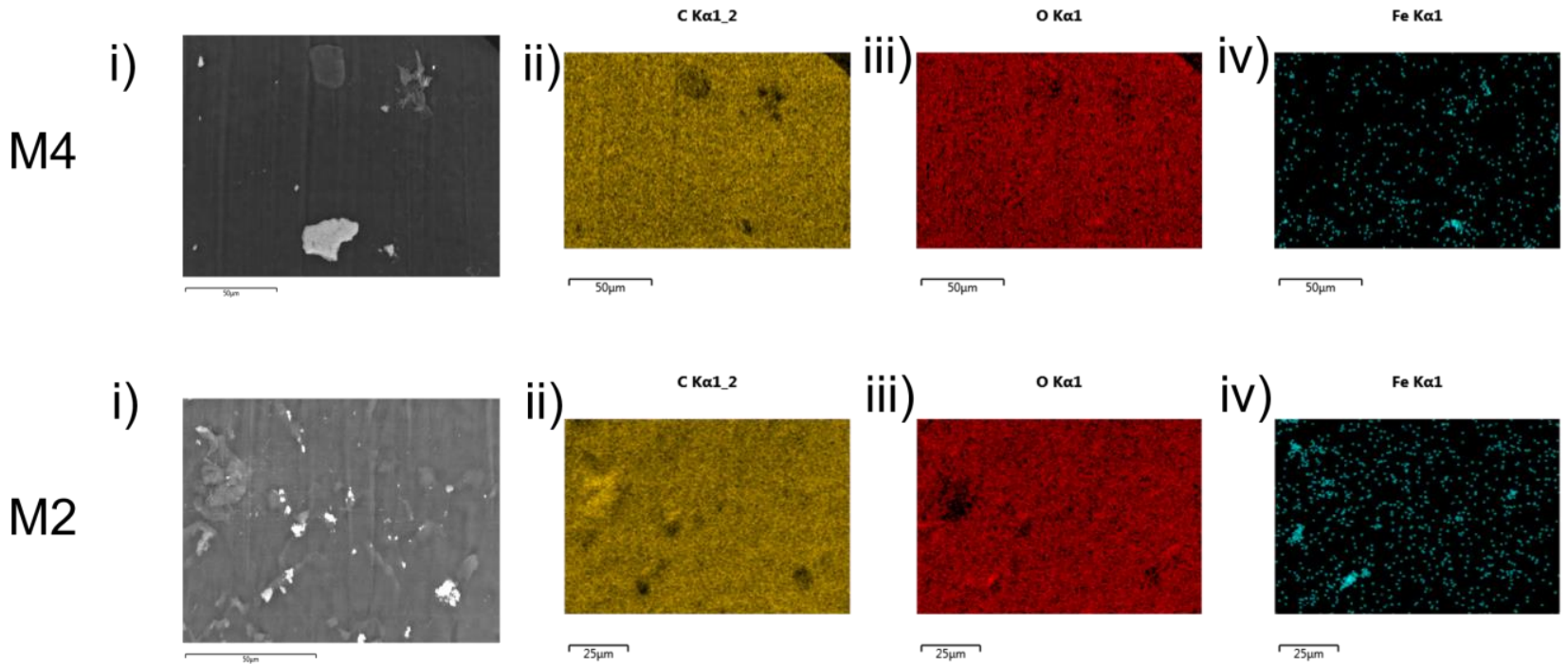

Fig. S5. SEM images of the $\varepsilon$-PCL polymeric scaffolds M2 and M4. i) Cross section of the biomaterial fiber. Bidimensional maps of the ii) carbon $(\mathrm{C})$, iii) oxygen $(\mathrm{O})$ and iv) iron (Fe) signal from the EDS analysis. 
a)

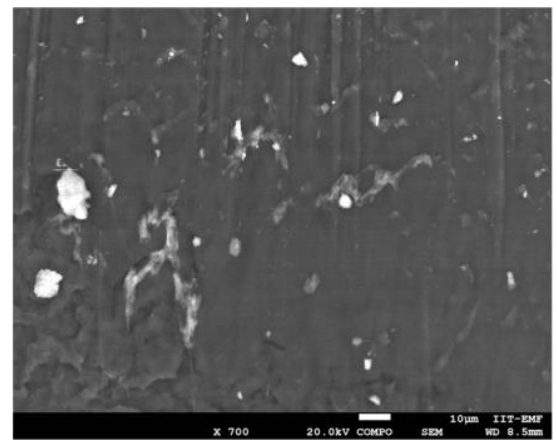

c)

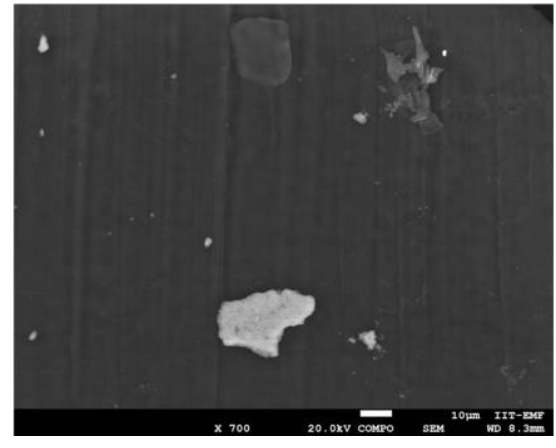

b)

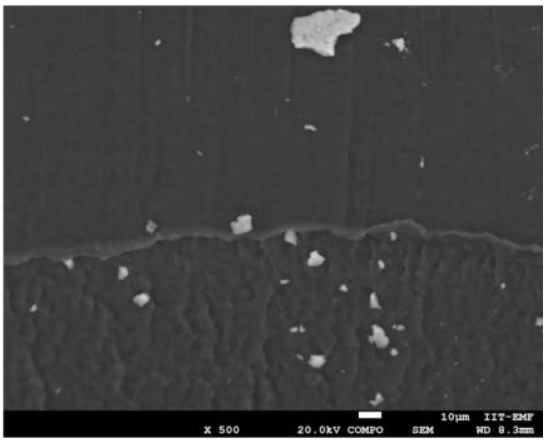

d)

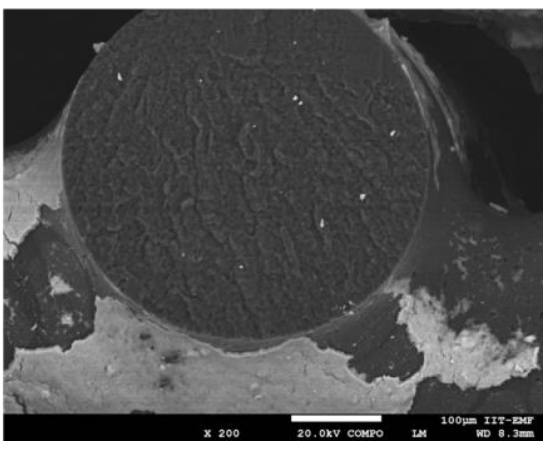

Fig. S6. Inside view of the polymeric scaffold and of the magnetic nanocrystals. A fiber-like structure is observed with presence of MNCs clusters, mostly present on the surface of the fiber.

SM5. Hyperthermia Treatment Outcome

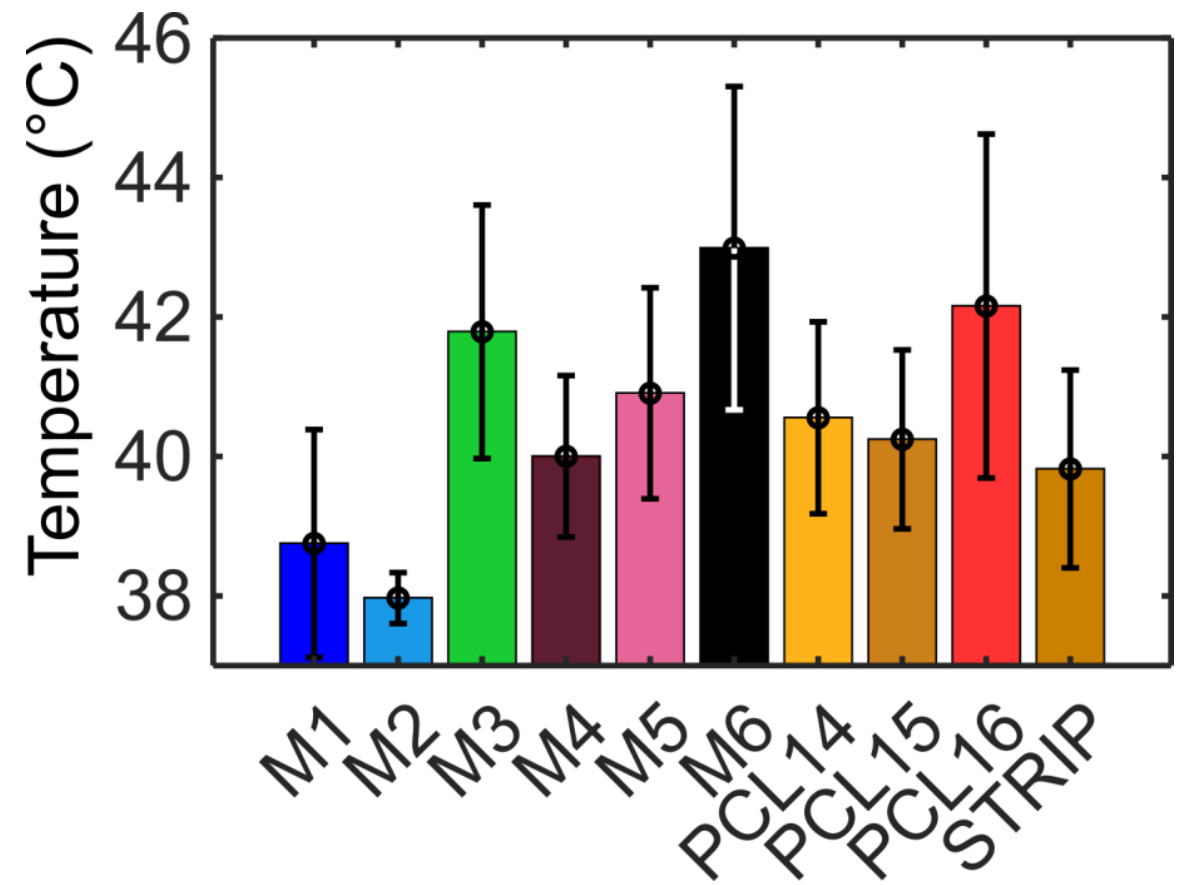

Fig. S7. T50 at $80 \mathrm{~min}$ in the tumor region, with standard deviation, for the ten drop-casted magnetic scaffolds 